\title{
Zampanolide binding to tubulin indicates crosstalk of taxane site with colchicine and nucleotide sites.
}

Jessica J. Field ${ }^{\dagger+}$, Benet Pera ${ }^{\ddagger^{+}}$, Juan Estévez Gallego ${ }^{{ }^{+}}$, Enrique Calvo ${ }^{\S}$, Javier RodríguezSalarichs $\$$, Gonzalo Sáez-Calvo ${ }^{\star}$, Didier Zuwerrall, Michel Jordi Prota $^{\nabla}$, Grégory Ménchon ${ }^{\nabla}$, John H. Miller ${ }^{\dagger}$, Karl-Heinz Altmann ${ }^{\|}$and J. Fernando Díaz ${ }^{*}$

${ }^{\dagger}$ Centre for Biodiscovery, School of Biological Sciences, Victoria University of Wellington, Wellington, New Zealand.

Centro de Investigaciones Biológicas (CIB), CSIC, Madrid, Spain.

${ }^{\S}$ Unidad de Proteómica, Centro Nacional de Investigaciones Cardiovasculares, Madrid, Spain.

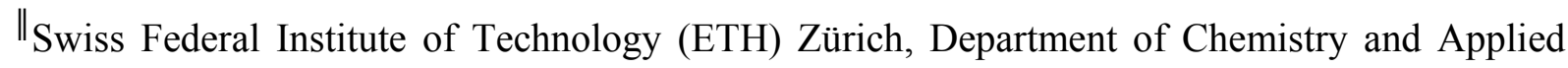
Biosciences, Institute of Pharmaceutical Sciences, HCI H405, Zürich, Switzerland.

${ }^{\nabla}$ Paul Scherrer Institut, Laboratory of Biomolecular Research, Villigen PSI, Switzerland.

Current addresses J.J Field, Amgen, 1120 Veterans Blvd, South San Francisco, CA 94080; B. Pera, Weill Cornell Medical College 1300 York Ave New York, NY 10065.

${ }^{+}$These authors contributed equally to this work.

*To whom correspondence should be addressed.

${ }^{\circ}$ Dedicated to Professor Susan Band Horwitz, of Albert Einstein College of Medicine, for her pioneering work on bioactive natural products. 


\section{ABSTRACT}

The marine natural product zampanolide and analogs thereof constitute a new chemotype of taxoid site microtubule-stabilizing agents with a covalent mechanism of action. Zampanolideligated tubulin has the switch-activation loop (M-loop) in the assembly-prone form and, thus, represents an assembly-activated state of the protein. In this study, we have characterized the biochemical properties of the covalently modified, activated tubulin dimer, and we have determined the effect of zampanolide on tubulin association and the binding of tubulin ligands at other binding sites. Tubulin activation by zampanolide does not affect its longitudinal oligomerization but does alter its lateral association properties. The covalent binding of zampanolide to $\beta$-tubulin affects both the colchicine site, causing a change of the quantum yield of a ligand bound, as well as the exchangeable nucleotide binding site, reducing the affinity for nucleotide. While these global effects do not change the binding affinity of 2-methoxy-5-(2,3,4trimethoxyphenyl)-2,4,6-cycloheptatrien-1-one (MTC) (a reversible binder of the colchicine site), the binding affinity of a fluorescent analog of GTP (Mant-GTP) at the nucleotide E-site is reduced from $12 \pm 2 \times 10^{5} \mathrm{M}^{-1}$ in the case of unmodified tubulin to $1.4 \pm 0.3 \times 10^{5} \mathrm{M}^{-1}$ in the case of the zampanolide tubulin adduct, indicating signal transmission between the taxane site and the colchicine and nucleotide sites of $\beta$-tubulin. 


\section{INTRODUCTION}

Microtubules are a component of the cytoskeleton that are formed by the non-covalent assembly of $\alpha, \beta$-tubulin heterodimers. They are highly dynamic entities that undergo continuous polymerization and depolymerization. Microtubules are most dynamic in mitosis and angiogenesis; thus, they are one of the most successful molecular targets for anticancer therapy ${ }^{1}$. The dynamic function of microtubules is targeted by a chemically diverse range of molecules with different binding sites on the microtubules. Microtubule-stabilizing agents (MSAs) bind to and stabilize microtubules, inhibiting the loss of the GDP-bound form of tubulin from the microtubule ends, resulting in mitotic arrest. This class of microtubule-targeting agents includes the clinically used taxanes $\left(\right.$ Taxol $^{\circledR}$, Taxotere ${ }^{\circledR}$ and Jevtana ${ }^{\circledR}$ ), the lactam analog of epothilone B (Ixempra $^{\circledR}$ ), and numerous novel compounds currently at various stages of preclinical and clinical development ${ }^{2}$. Although taxanes have been highly successful in treating cancer, their low solubility, toxicity, and susceptibility to multiple drug resistance imposes serious limits on their use. Thus, there is a need for new MSAs that overcome these limitations. As a possible alternative, mitotic kinases have recently become attractive targets for anticancer drug development, given their essential role in mitosis. However, clinical data on these kinase inhibitors have not been encouraging, with many outcomes only characterized as 'stable disease ${ }^{3}$. This is most likely due to up-regulation of other kinases or pathways to compensate for those inhibited by the drugs ${ }^{4}$. However, there are no cellular substructures other than microtubules that can segregate chromosomes.

Therefore, resistance to MSAs does not arise from activation of alternative pathways, but is mainly due to over-expression of efflux pumps ${ }^{5}$ and the up-regulation of the BIII tubulin isoform $^{6}$. In addition, microtubule targeting agents not only target mitotic cells but also 
interphase cells by inhibiting, for example, such functions as microtubule trafficking ${ }^{2}$. Thus, microtubules continue to be one of the most attractive anticancer targets, especially since many newly developed MSAs are not substrates of the P-gp efflux pump and/or are not affected by upregulation of the $\beta I I I$ isoform of tubulin ${ }^{7}$. Furthermore, there are several covalent microtubuledestabilizing agents $(\mathrm{MDAs})^{8}$ and three MSAs that can overcome the overexpression of drug efflux pumps ${ }^{9-11}$ due to covalent binding to tubulin. The latter would also be predicted not to be affected by changes in tubulin isotype expression. Covalent binding produces a prolonged interaction with the drug target even though residual, free drug is cleared from the system. This may lead to a more desirable clinical profile since less frequent dosing and lower drug concentrations can be used ${ }^{12}$. This concept, however, has not yet been clinically employed with drugs interacting with the tubulin/microtubule system.

(-)-Zampanolide (Figure 1) is a marine macrolide that binds to the taxoid binding site on $\beta$-tubulin in a covalent fashion, in contrast to traditional MSAs, which are all reversible binders $^{10,13,14}$. Covalent attachment of zampanolide to $\beta$-tubulin can occur at two specific amino acids, Asn228 and His229, but the major pathway involves reaction with the His229 side chain ${ }^{10}$, an important residue in the taxoid binding site s. $^{15,16}$.

To meet the quest for more effective drugs with a MSA mechanism of action, it is of utmost importance to understand the mechanism that these compounds use to stabilize microtubules. Two different mechanisms have been proposed to account for the stabilization effect of paclitaxel site ligands on microtubules. Prota et $\mathrm{al}^{17}$ have observed that epothilone A or zampanolide binding to the site induces the structuring of the M-loop, an important secondary structural element involved in stabilization of the microtubule lattice by increasing lateral protofilament interactions ${ }^{18}$. The M-loops of both tubulin subunits in the dimer are thought to 
have a pre-disposition to form a helical structure, which is a prerequisite for efficient lateral contacts between protofilaments upon polymerization into microtubules ${ }^{17}$. The helical structuring of the M-loop is proposed to lower the energy needed for the curved-to-straight conformational change that occurs when tubulin is incorporated into the polymerizing microtubules. The binding of an MSA to the tubulin dimer either at the taxoid site or at the laulimalide/peloruside site $^{19}$, which is distinct from the taxoid site, causes structural organization of the M-loop according to the requirements for microtubule assembly, promoting the incorporation of the dimer into the microtubule lattice. In this way, the presence of an MSA reduces the entropy loss compared to assembly in the absence of an MSA ${ }^{17}$.

As an alternative hypothesis, Amos and Löwe ${ }^{20}$ and Nogales et al. ${ }^{21}$ proposed that paclitaxel inhibits a series of destabilizing conformational changes in the microtubule that allosterically induce a conformation in $\beta$-tubulin that is similar to that of the GTP-bound state. Using cryo-electron microscopy of dynamic microtubules and microtubules stabilized by GMPCPP (a slowly hydrolysable GTP analog) or by paclitaxel, it was determined that nucleotide hydrolysis leads to a compaction around the E-site at the longitudinal interfaces that is inhibited by paclitaxel. Moreover, no change was detected in the lateral interfaces, and thus it was proposed that the paclitaxel effect is exerted through the nucleotide site in the longitudinal region and requires signal transmission between both binding sites ${ }^{21}$. A more recent cryo-electron microscopy study of paclitaxel and zampanolide induced microtubules ${ }^{22}$ indicates that binding of these drugs to 13-protofilament microtubules results in a similar modification in the flexibility of the lateral contacts of the protofilaments, both inducing lattice heterogeneity despite having different binding modes to the taxane pocket. 
Although a conformational change at the lateral interface is clearly observed in the structures of zampanolide-ligated tubulin ${ }^{17}$, no changes or very minor changes are seen in the nucleotide binding site of the ligated dimer, nor is there a visible structural effect on the GTP and GDP bound to tubulin ${ }^{17}$. However, if binding to the taxane site exerts its effect through the longitudinal interface, it should somehow mimic the effect of the nucleotide in the longitudinal and lateral interfaces, facilitating the curved to straight transition. This structural effect on the microtubule could result from signal transmission between the paclitaxel site and the E-site.

In the present study, we investigated the biochemistry of the zampanolide-ligated, activated form of tubulin (termed the 'zampanolide-adduct') in order to obtain information about the changes in tubulin induced by the activation process.

\section{RESULTS AND DISCUSSION}

Oligomerization properties of the zampanolide-adduct: Analytical ultracentrifugation was used to determine the sedimentation velocity profiles of unmodified and zampanolidemodified tubulin (the zampanolide-adduct) under different conditions. Analytical ultracentrifugation profiles are represented by the sedimentation coefficient $\mathrm{S}$ (which is related to the size of the species) vs the concentration of protein. The tubulin dimer peak can be observed at approximately $5.8 \mathrm{~S}$, while tubulin oligomers and larger aggregates, sediment at higher $\mathrm{S}$. The type of tubulin oligomers produced is dictated by the concentration of $\mathrm{Mg}^{2+}$. Solutions of tubulin (with either GTP or GDP bound) with low concentrations of free $\mathrm{Mg}^{2+}(\sim 1 \mathrm{mM})$ contain only the $\alpha, \beta$-heterodimer (5.8 S). Moderately higher amounts of $\mathrm{Mg}^{2+}(3-5 \mathrm{mM})$ result in the formation of small tubulin oligomers; at high concentrations of $\mathrm{Mg}^{2+}(>10 \mathrm{mM})$ in GDP buffer, tubulin 
double rings are formed (see Figure $12 \mathrm{in}^{23}$ for a schematic of $\mathrm{Mg}^{2+}$-induced self-association of tubulin).

At very low $\mathrm{Mg}^{2+}$ concentrations $\left(0.028 \mathrm{mM}\right.$ free $\mathrm{Mg}^{2+}$ for $0.1 \mathrm{mM}$ GTP buffer and 0.039 $\mathrm{mM}$ free $\mathrm{Mg}^{2+}$ for $0.1 \mathrm{mM}$ GDP buffer), both unmodified and zampanolide-ligated tubulin are unable to oligomerize and only dimers are observed (Figure 1A-1B, respectively). At a slightly higher concentration of free $\mathrm{Mg}^{2+}(0.14 \mathrm{mM})$, a small proportion of oligomers was observed at 9 $\mathrm{S}$, but again no significant differences were observed between the unmodified protein and the adduct, thus indicating that the binding of zampanolide had no notable effect on the longitudinal tubulin-tubulin interactions responsible for the early aggregation events (Figure 1C). Under conditions with moderate concentrations of free $\mathrm{Mg}^{2+}(0.77 \mathrm{mM})$ and in the absence of zampanolide, tubulin dimers, a small proportion of tetramers, and some larger oligomers were observed. In contrast, with the zampanolide-adduct, the amount of free tubulin dramatically decreased, and the small oligomers disappeared, indicating that the adduct readily assembles into microtubules as is shown by electron microscopy under these conditions (Figure 1D). The same effect was seen (for unmodified tubulin) in the presence of excess epothilone B (Figure 1E). The data show that the zampanolide-adduct is more prone to polymerize into microtubules than unligated-tubulin, presumably due to the stabilization of the lateral interdimer contacts that are essential for microtubule formation from oligomers.

These findings were also confirmed in GDP buffer (Figure 2). At high $\mathrm{Mg}^{2+}$ concentrations (3.31 mM free), the zampanolide-adduct assembled more readily than unmodified tubulin which is mainly in the form of dimers of dimers (tetramers), although other oligomers are observed both in the presence and in the absence of zampanolide. 

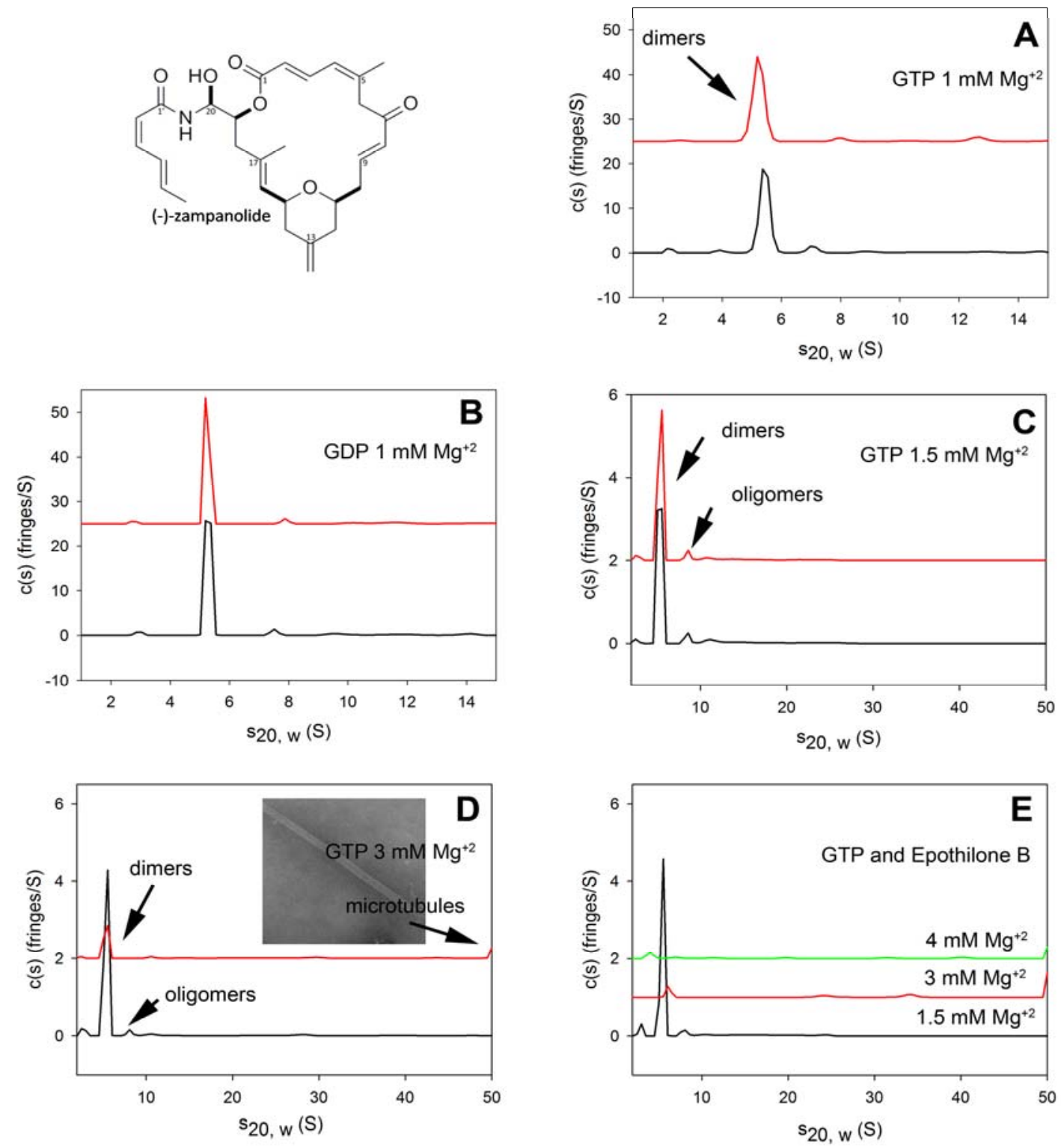

Figure 1.- Effect of adduct formation and $\mathrm{Mg}^{2+}$ on tubulin self association analyzed by sedimentation velocity. Structure of (-)-zampanolide. Sedimentation coefficient distribution c(s) of $50 \mu \mathrm{M}$ tubulin with $55 \mu \mathrm{M}$ zampanolide (red line) or DMSO (black line) with A. $0.1 \mathrm{mM}$ GTP, low free $\mathrm{Mg}^{2+}\left(1 \mathrm{mM}\right.$ total $\mathrm{Mg}^{2+}, 0.028 \mathrm{mM}$ free $\left.\mathrm{Mg}^{2+}\right)$ and B. $0.1 \mathrm{mM}$ GDP, low free $\mathrm{Mg}^{2+}\left(1 \mathrm{mM}\right.$ total $\mathrm{Mg}^{2+}, 0.039 \mathrm{mM}$ free $\left.\mathrm{Mg}^{2+}\right)$ C. $0.1 \mathrm{mM}$ GTP, low concentration of free $\mathrm{Mg}^{2+}$ (1.5 mM total $\mathrm{Mg}^{2+}, 0.14 \mathrm{mM}$ free $\mathrm{Mg}^{2+}$ ). D. $0.1 \mathrm{mM}$ GTP moderate free $\mathrm{Mg}^{2+}$ concentrations (3 $\mathrm{mM}$ total $\mathrm{Mg}^{2+}, 0.77 \mathrm{mM}$ free $\mathrm{Mg}^{2+}$ ) Inset Electron micrography of the sample in the presence of zampanolide. E. Sedimentation coefficient distribution c(s) of $50 \mu \mathrm{M}$ tubulin with $100 \mu \mathrm{M}$ epothilone B and $0.1 \mathrm{mM}$ GTP at different concentrations of $\mathrm{Mg}^{2+} 1.5 \mathrm{mM}$ total $\mathrm{Mg}^{2+}(0.14 \mathrm{mM}$ free, black line), $3 \mathrm{mM} \mathrm{Mg}^{2+}\left(0.77 \mathrm{mM}\right.$ free, red line), $4 \mathrm{mM} \mathrm{Mg}^{2+}$ (1.31 $\mathrm{mM}$ free, green line). The velocities observed at $>50 \mathrm{~S}$ are large assemblies (microtubules are observed in the electron micrographs as is shown in the inset of panel D) 

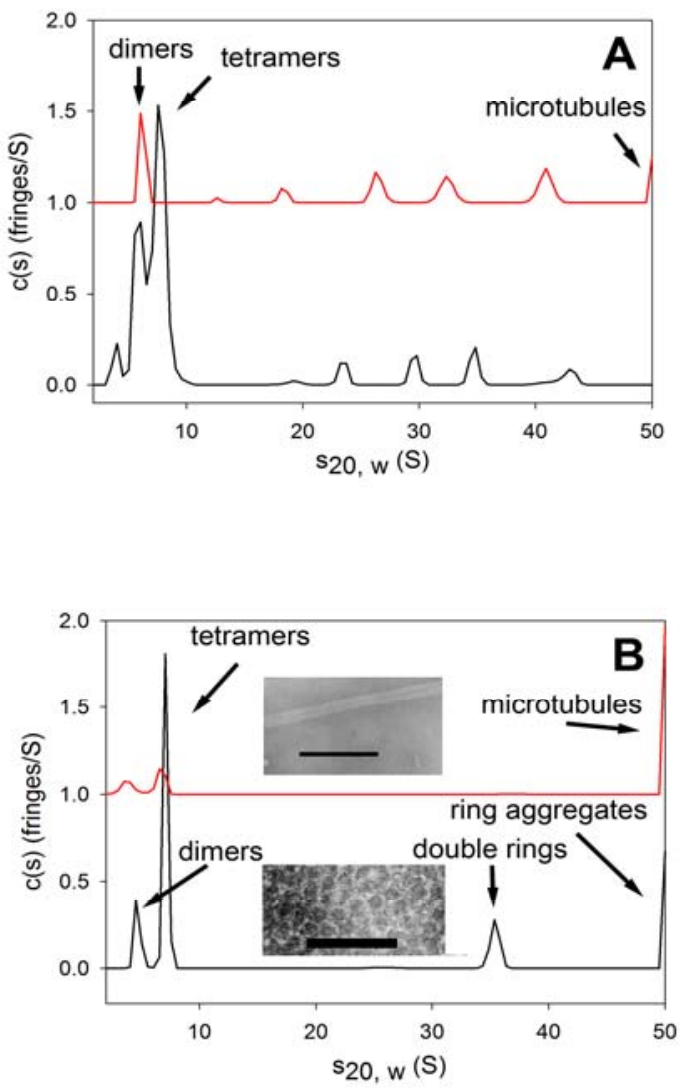

Figure 2.- Effect of adduct formation and $\mathrm{Mg}^{2+}$ on tubulin self association. Sedimentation coefficient distribution c(s) of $50 \mu \mathrm{M}$ tubulin with $55 \mu \mathrm{M}$ zampanolide (red line) or DMSO (black line) A. In the presence of $1 \mathrm{mM}$ GDP and $1.42 \mathrm{mM}$ free (4 mM total) $\mathrm{Mg}^{2+}$. B. In the presence of $1 \mathrm{mM}$ GDP and 3.31 $\mathrm{mM}$ free $\left(7 \mathrm{mM}\right.$ total) $\mathrm{Mg}^{2+}$. Species at $6 \mathrm{~S}$ are tubulin dimers, Species at $8 \mathrm{~S}$ are dimers of dimers. Species at $36 \mathrm{~S}$ are double rings as confirmed by electron microscopy, while other intermediate peaks are uncharacterized oligomers. The velocities observed at $>50 \mathrm{~S}$ are large assemblies (insets: microtubules are observed in the electron micrographs, in the presence of zampanolide while double ring arrays are observed in its absence bar represents $200 \mathrm{~nm}$ ).
As previously shown ${ }^{24,25}$, in GDP buffer with a high concentration of free $\mathrm{Mg}^{2+}(6.78 \mathrm{mM})$, unmodified tubulin forms characteristic double rings (peak at 35$41 \mathrm{~S}$ ) and double ring aggregates (over 50S). Strikingly, however, under the same conditions the zampanolide-ligated tubulin, regardless of the bound nucleotide, completely assembled into microtubules as checked by electron microscopy with no double rings observed, since the concentration was well above the $\mathrm{Cr}$ required for assembly.

Zampanolide does not alter the affinity of peloruside and colchicine for their binding sites: One important unresolved issue in the field of microtubule-ligand interactions is the communication between different binding sites, a process that can potentially lead to synergy between drugs ${ }^{19}$. To study this cross-talk, we measured the binding constant of the zampanolide-adduct or microtubules assembled from the adduct for other ligands. More specifically, we measured the binding affinities of peloruside A and of the fluorescent colchicine analog MTC for tubulin. 
The binding constant for peloruside $\left(\mathrm{K}_{\mathrm{b}}\right)$ with unmodified, stabilized microtubules at $25^{\circ} \mathrm{C}$ was found to be $4.0 \pm 0.3 \times 10^{6} \mathrm{M}^{-1}$ (standard error of three independent measurements), which is similar to the values previously determined ${ }^{26}$. This affinity was not significantly different for zampanolide-modified, stabilized microtubules $\left(\mathrm{Kb}=3.1 \pm 0.4 \times 10^{6} \mathrm{M}^{-1}\right)$ (standard error of three independent measurements), thus indicating that the zampanolide modification had no effect on the structure of the laulimalide/peloruside site.

The binding interaction of MTC with tubulin was assessed by adding increasing concentrations of MTC to a constant concentration of unmodified tubulin or the zampanolideadduct $(10 \mu \mathrm{M})$ (in the absence of added $\mathrm{Mg}^{2+}$ the adduct is in the form of dimers), and the fluorescence of MTC was then used to calculate the binding constant. MTC fluorescence saturated at approximately $10 \mu \mathrm{M}$ for both tubulin variants, indicating a 1:1 stoichiometry for binding of MTC to both unmodified tubulin and the zampanolide-adduct (Figure 3A). Thus, zampanolide modification of tubulin did not affect the stoichiometry of binding of MTC to the colchicine site; however, the fluorescence of MTC was significantly higher for binding to the adduct compared to unmodified tubulin. These experiments revealed that the affinity of MTC for the site was unchanged upon the formation of the zampanolide-adduct (Figure 3B), as Kb values $\left(4.5 \pm 0.1 \times 10^{5} \mathrm{M}^{-1}\right.$ standard error of three independent measurements) were similar to those previously determined in our laboratory for unmodified tubulin $\left(4.7 \pm 0.3 \times 10^{5} \mathrm{M}^{-1}\right)^{27}$.

The difference in fluorescence between MTC bound to the zampanolide adduct and unmodified tubulin was similar to the increase in fluorescence that is seen when $\mathrm{Mg}^{2+}$ is added to a MTC-tubulin complex prepared in a $\mathrm{Mg}^{2+}$ free buffer. This increase in MTC fluorescence has been proposed to involve changes in the microenvironment upon high affinity $\mathrm{Mg}^{2+}$ binding to the $\alpha$-subunit ${ }^{28}$. 

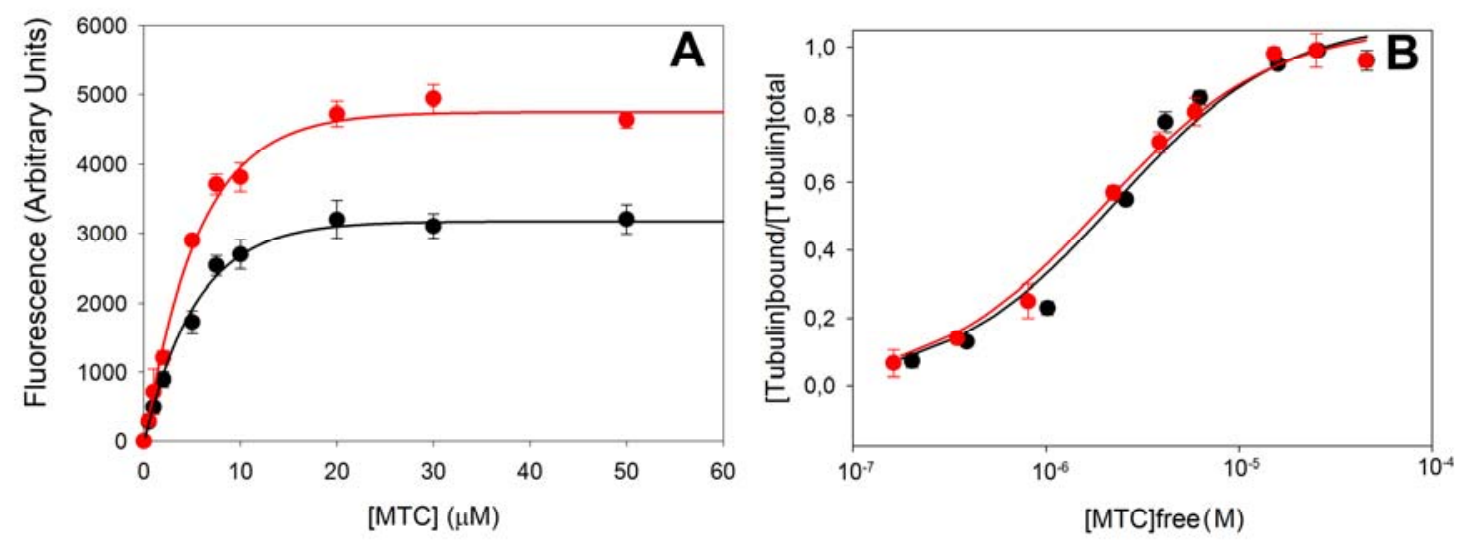

Figure 3.- The effect of adduct formation on the affinity of the colchicine binding site for

MTC. A. Fluorescence (relative fluorescence units) of increasing concentrations of MTC (a fluorescent colchicine analog) when binding to $10 \mu \mathrm{M}$ zampanolide-adduct (red) or unligated tubulin (black). B. Binding curves of MTC to the zampanolide-adduct (red) or unligated tubulin (black). Error bars are the standard errors of 5 independent measurements.

However, an alternative explanation could be that a slight change in the configuration of the colchicine binding site, either upon addition of $\mathrm{Mg}^{2+}$ or when zampanolide is pre-bound to tubulin, leads to the increased fluorescence.

Crystal structure of the tubulin-MTC and tubulin-MTC-zampanolide complexes: In order to understand the observed higher fluorescence of MTC for binding to the adduct compared to unmodified tubulin, we solved the structures of both MTC and the binary MTC/zampanolide complex with tubulin by X-ray crystallography. 
A

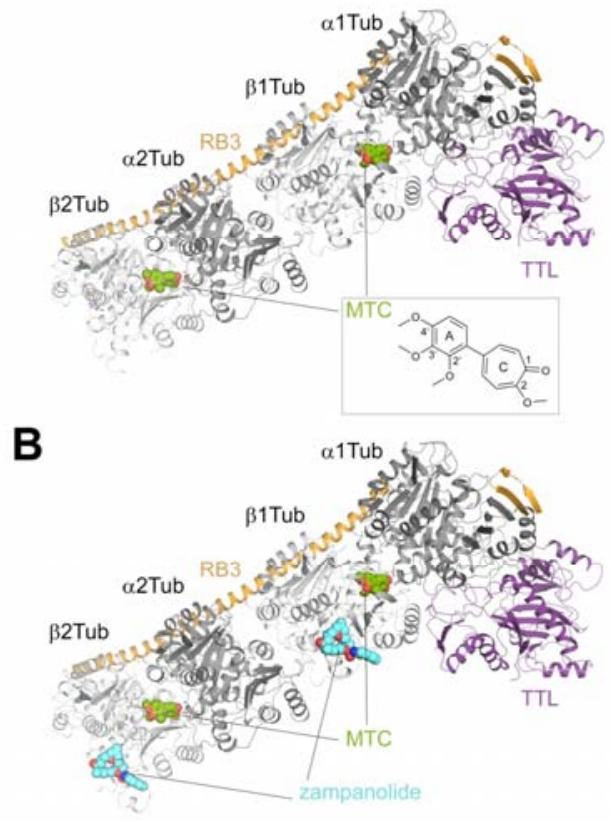

C

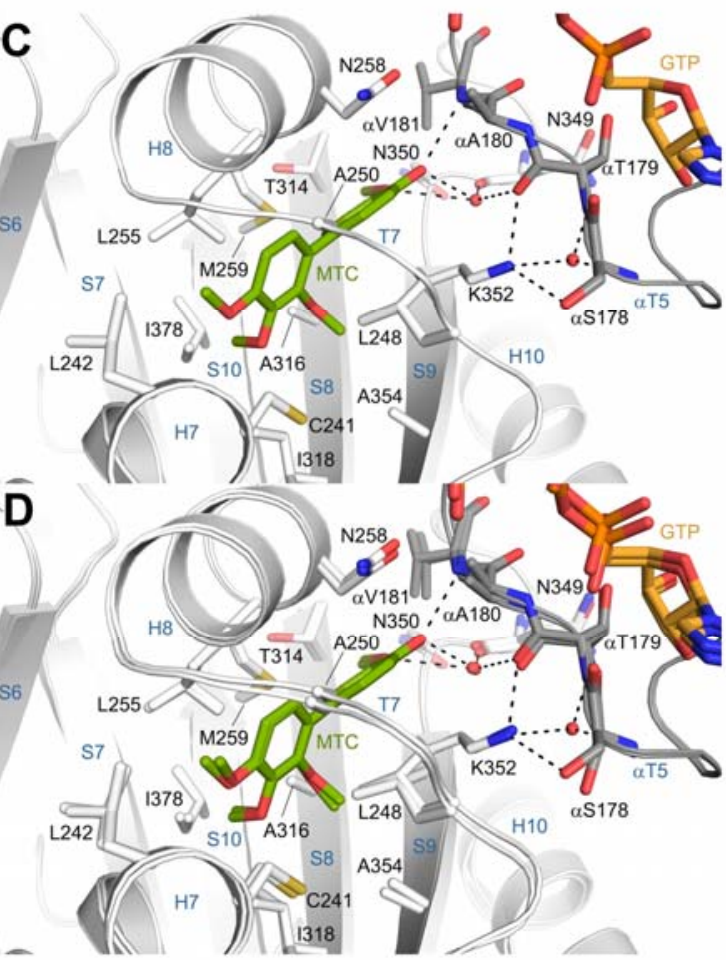

Figure 4. Overall structures of both the $\mathbf{T}_{2} \mathbf{R}$-TTL-MTC and $\mathbf{T}_{2} \mathbf{R}$-TTL-MTC-zampanolide complexes. Overall views of (A) the $\mathrm{T}_{2} \mathrm{R}$-TTL-MTC and (B) the $\mathrm{T}_{2} \mathrm{R}$-TTL-MTC-zampanolide complex structures. The $\alpha$-tubulin and $\beta$-tubulin chains are shown in dark and light grey, TTL is shown in violetpurple and RB3 is in orange ribbon representation, respectively. The tubulinbound MTC and zampanolide molecules are represented as green and cyan spheres, respectively. The chemical structure of MTC is displayed in the dotted box. X-Ray analysis of the tubulin-MTC complex. (C) Close-up view of the interaction network observed between MTC (green) and tubulin (gray). Interacting residues of tubulin are shown in stick representation and are labeled. Oxygen and nitrogen atoms are colored red and blue, respectively; carbon atoms are in green (MTC) or gray (tubulin). Hydrogen bonds are depicted as black dashed lines. Secondary structural elements of tubulin are labeled in blue. (grey ribbon;PDB ID 5NFZ). For simplicity, only $\alpha$-tubulin residues are indicated with an $\alpha$ with $\beta$-tubulin as the default tubulin.. (D) Superposition of both MTC-bound sites in chain B and D in the same orientation and representation as in (C). 
Table 1. Data collection and refinement statistics.

$\mathrm{T}_{2} \mathrm{R}$-TTL-MTC

$\mathrm{T}_{2} \mathrm{R}$-TTL-MTC-zampanolide

\section{Data collection $^{\mathrm{a}}$}

Space group

$\mathrm{P} 2{ }_{1} 2_{1} 2_{1}$

$\mathrm{P} 2{ }_{1}{ }_{1} 2_{1}$

Cell dimensions

$$
a, b, c(\AA)
$$

$105.2,158.8,180.4$

$104.2,157.4,178.9$

Resolution $(\AA)$

$59.78-2.10(2.15-2.10)$

$47.69-2.20(2.26-2.20)$

$\mathrm{R}_{\text {meas }}(\%)$

$14.0(300.5)$

$11.6(222.6)$

$\mathrm{R}_{\mathrm{pim}}(\%)$

$4.2(87.9)$

$4.6(92.2)$

$\mathrm{CC}_{1 / 2}{ }^{\mathrm{b}}$

$99.9(45.2)$

$99.9(29.1)$

$\mathrm{I} / \sigma \mathrm{I}$

$16.2(0.9)$

$13.4(0.9)$

Completeness (\%)

$100.0(99.8)$

$100.0(99.8)$

Redundancy

$13.5(10.6)$

$6.7(6.5)$

\section{Refinement}

Resolution $(\AA)$

$59.78-2.10$

$47.69-2.20$

No. unique reflections

175862

149309

$\mathrm{R}_{\text {work }} / \mathrm{R}_{\text {free }}(\%)$

$18.2 / 21.8$

$17.3 / 22.0$

Average B-factors $\left(\AA^{2}\right)$

Complex

Solvent

Ligand (MTC, chain B/D)

Ligand (zampanolidechain B/D)
60.5

51.9

$41.6 / 44.3$

-1 -

42.3

Wilson B-factor

Root mean square deviation from ideality
Bond length $(\AA)$
0.007
0.008
Bond angles $\left(^{\circ}\right)$
0.856
0.957

Ramachandran statistics ${ }^{c}$
Favored regions (\%)
98.0
97.0
Allowed regions (\%)
2.0
2.9
Outliers (\%)
0
0.1

55.9

$58.2 / 56.3$

$64.6 / 60.4$

${ }^{a}$ Highest shell statistics are in parentheses. ${ }^{b} \mathrm{CC} 1 / 2=$ percentage of correlation between intensities from random half-datasets ${ }^{58} .{ }^{\mathrm{c}}$ As defined by MolProbity ${ }^{56}$. 
To this end, we soaked MTC alone or together with zampanolide into crystals formed by a protein complex composed of two $\alpha \beta$-tubulin heterodimers, the stathmin-like protein RB3 and tubulin tyrosine ligase $\left(\mathrm{T}_{2} \mathrm{R}-\mathrm{TTL}\right)^{17,29}$ (although a 10 times higher concentration of MTC was needed to soak the ligand in the presence of zampanolide). Using this approach, both the tubulinMTC and the tubulin-MTC-zampanolide structures were determined to 2.1 and $2.2 \AA$ resolution, respectively (Figure 4AB; Table 1). The overall structure of tubulin in both the $\mathrm{T}_{2} \mathrm{R}$-TTL-MTC and the T2R-TTL-MTC-zampanolide complexes superimposed well with the one obtained in the absence of any ligand (1) (rmsdT2R-TTL-MTC of $0.41 \AA \AA$ over $2042 \mathrm{C} \alpha$-atoms; rmsd T2R-TTLMTC-zampanolide of $0.46 \AA$ over $2037 \mathrm{C} \alpha$-atoms), suggesting that binding of both ligands does not affect the overall conformation of tubulin. MTC binds to the colchicine site of tubulin, which is formed by residues of strands $\mathrm{S} 8, \mathrm{~S} 9$ and $\mathrm{S} 10$, loop $\mathrm{T} 7$, and helices $\mathrm{H} 7$ and $\mathrm{H} 8$ of $\beta$-tubulin, and loop T5 of $\alpha$-tubulin (Figure 4CD). In the tubulin-MTC complex, the 2',3',4'trimethoxyphenyl substituted A ring of MTC is buried in the pocket shaped by $\beta C y$ s 241 , $\beta$ Leu242, $\beta$ Leu248, $\beta$ Ala250, $\beta$ Leu255, $\beta$ Asn258, $\beta$ Met259, $\beta$ Ala316, $\beta$ Ile318, $\beta$ Ala354, and BIle378 (Figure 4C). The 2-methoxy substituted cycloheptatrien-1-one (C ring) of MTC is stacked between $\beta$ Asn 258 and $\beta$ Lys352, and is further stabilized by hydrophobic interactions to $\beta$ Met $259, \beta$ Thr314, and $\beta$ Ala316, and by the T5 loop residues $\alpha$ Ala180 and $\alpha$ Val181. In addition, one hydrogen bond is formed between the 1-carbonyl of MTC and the main chain amide of $\alpha$ Val181. Furthermore, both the 1-carbonyl and 2-methoxy groups form watermediated hydrogen bonds to the main chain carbonyls of $\beta$ Asn349 and $\alpha$ Thr 179 . This binding mode is maintained in both the occupied binding sites in our crystal system (Figure 4D; RMSD chain D onto chain B $0.29 \AA$ over $377 \mathrm{C} \alpha$-atoms). This arrangement together with the hydrogen bonds formed by $\beta$ Lys352 to the main chain carbonyl of $\alpha$ Thr179 and to $\alpha$ Ser178 allows for a 
stabilization of the $\alpha \mathrm{T} 5$ loop in the same conformation as observed for colchicine (Figure 5A; PDB ID 4O2B). Comparison of the tubulin structure in the bound and unbound states reveals similar conformational adaptations of loops T7 and T5 of $\beta$ - and $\alpha$-tubulin upon ligand binding, as reported for other colchicine-site ligands (Figure 5B; PDB ID 4I55).

In the tubulin-MTC-zampanolide complex, both ligands are bound in both the chains B and D (Figure 4B). Compared to the tubulin-MTC complex, no major structural changes were observed in the colchicine site of the binary complex (Figure 5C), except for a backbone shift and reorientation of the $\beta$ Ala250 methylene on the T7-loop (1.6 $\AA$ shift of backbone; $2.9 \AA$ shift of the $C \beta$ ), this change of the orientation points the $\beta$ Ala 250 methylene to the trimethoxyphenyl ring of MTC. The analysis suggests that the covalent binding of zampanolide on tubulin does not affect the binding mode of the MTC compound in our crystal system, but the environment of the trimethoxyphenyl ring is changed, justifying the change in fluorescence observed. However, since both the binding sites are connected through the core helix $\mathrm{H} 7^{30}$ (Figure 5C), the presence of zampanolide bound to $\beta$ His229 in the binding site could also change the dynamicity of this helix and may result in a stabilization of the MTC molecule in the "high fluorescence" state observed in the crystal structure. This could also explain the measured change in fluorescence.

Zampanolide reduces the affinity of nucleotides for the E-site:Finally, we examined the effect of zampanolide-adduct formation on the E-site (which is closer to the taxoid site than any of the other binding sites). A tubulin heterodimer binds two molecules of a guanosine nucleotide $^{31}$, one non-exchangeable, non-hydrolysable GTP molecule tubulin is in the dimeric state, both bound nucleotides are generally GTP, but soon after 
A

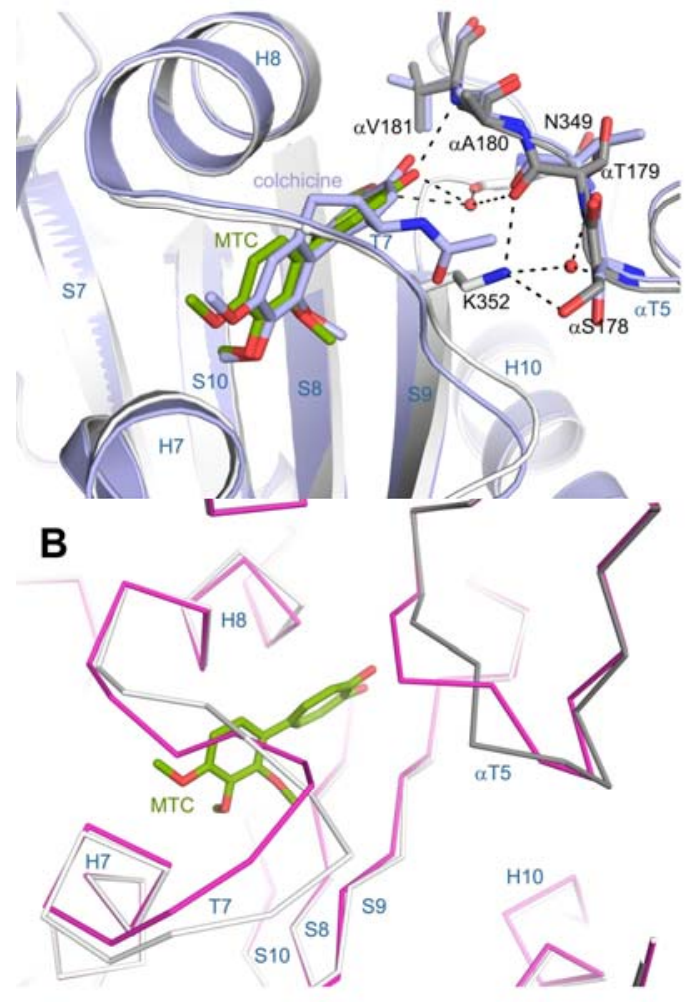

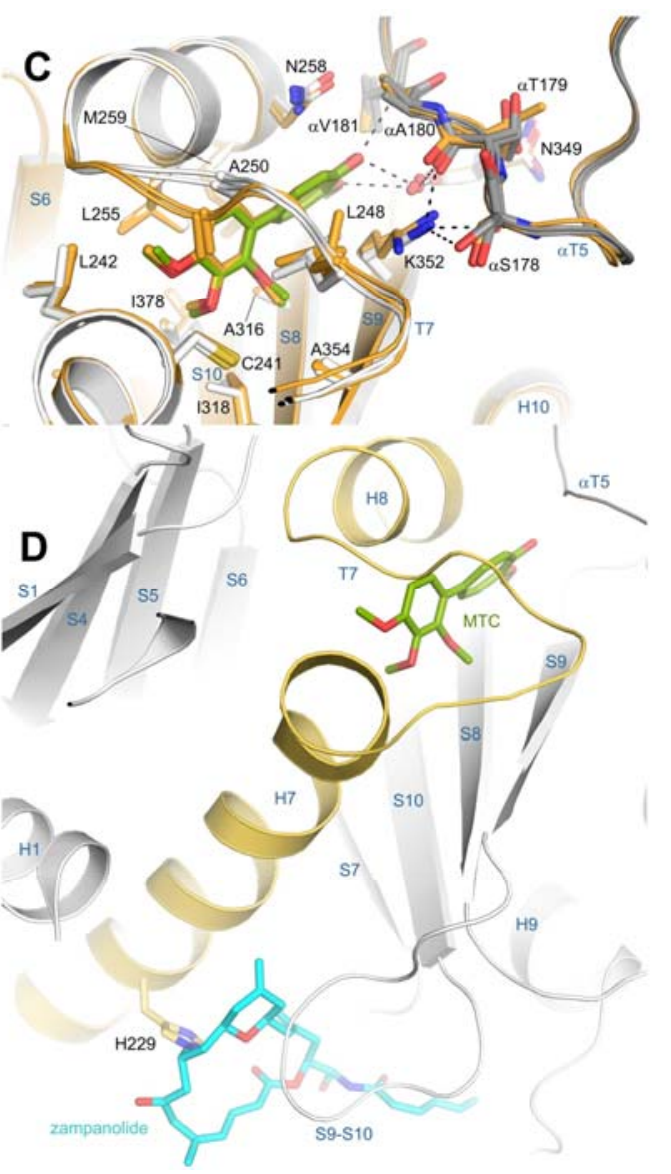

Figure 5. Comparison of MTC and colchicine tubulin-binding modes. (A) Superimposition of the tubulin-MTC (green/grey) and tubulin-colchicine (light blue; PDB ID 4O2B) complex structures in the same orientation and representation as in Figure 4C. (B) Ribbon representation of the superimposed apo (magenta, PDB ID 4I55) and tubulin bound MTC (light and dark grey) structures highlighting the conformational changes that occur upon ligand binding at both the $\alpha \mathrm{T} 5$ and $\beta$ T7-loops. The secondary structural elements are labeled in blue. The MTC is in green sticks representation. The structures were superimposed onto their $\beta 1$-tubulin chains (chain $B$ of $T_{2} R$ TTL, rmsd of $0.17 \AA$ over $336 \mathrm{C}_{\alpha}$-atoms). Comparison of both the $\mathbf{T}_{2} \mathbf{R}$-TTL-MTC and $\mathbf{T}_{2} \mathbf{R}$ TTL-MTC-zampanolide complexes. (C) Superimposition of the MTC binding sites (chains B and D) of both the tubulin-MTC (green/grey) and tubulin-zampanolide-MTC (orange, PDB ID 5NG1) complex structures in the same representation as in Figure 5C. (D) Ribbon representation of simultaneous MTC- and zampanolide-binding to $\beta$-tubulin. The ligands are in green (MTC) and cyan (zampanolide) stick representation, respectively. The core helix H7, which connects the two binding sites, together with the loop T7 and helix H8 are highlighted in yellow-orange. 
assembly into microtubules, the GTP at the E-site is hydrolyzed to GDP. Thus, assembled microtubules contain $1 \mathrm{~mol}$ of GDP and $1 \mathrm{~mol}$ of GTP per tubulin heterodimer. Since the side chain of Asn228 faces the E-site and interacts with the bound nucleotide, and since zampanolideadduct formation involves the covalent attachment of zampanolide to the neighboring amino acid His229, it was hypothesized that the zampanolide-adduct could exhibit an altered binding affinity of the E-site for guanosine nucleotides. A comparison was therefore made between the nucleotide content of the zampanolide-adduct with that of unmodified tubulin under a set of different conditions. The results indicate that formation of the zampanolide-adduct causes a significant decrease in the amount of bound nucleotide in the E-site of dimeric tubulin (Table 2),

Table 2 - Loss of nucleotide binding affinity upon covalent modification of the taxoid binding site.

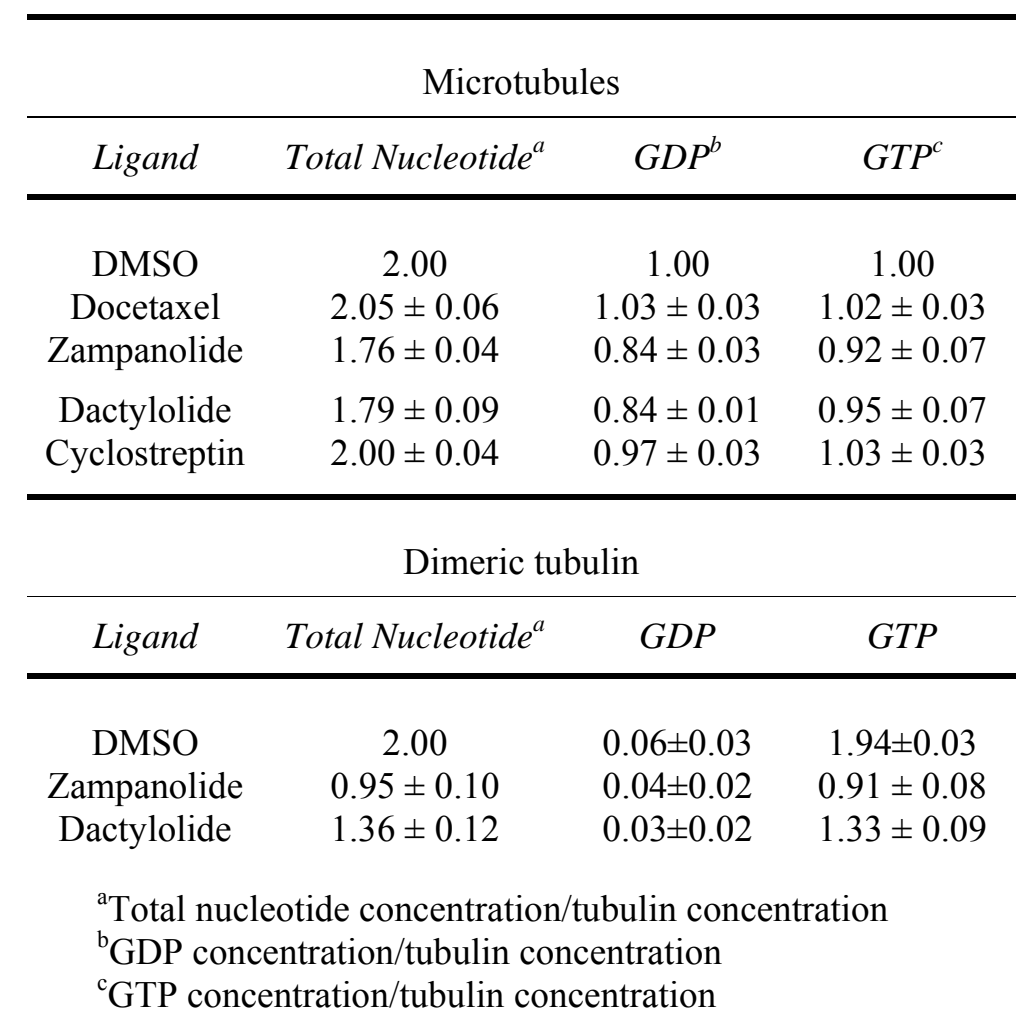


with a complete loss of E-site bound nucleotide being observed when the protein is passed through a G-25 column in the presence of $10 \mu \mathrm{M}$ nucleotide. This indicates a significant decrease in binding affinity compared with the unmodified protein, which retains the nucleotide under this condition. For comparison, in previous studies with cyclostreptin, the latter caused a $20 \%$ loss of nucleotide in dimeric tubulin, while neither DMSO nor docetaxel showed any effect on the nucleotide content in microtubules or dimeric tubulin (Table 2). The effect is not due to an inactivation of the site, since the adduct incubated in $3.4 \mathrm{M}$ glycerol, $10 \mathrm{mM}$ sodium phosphate, 1 mM EDTA, 6 mM MgCl 2 , pH 6.7 plus 0.1 mM GTP (Gycerol Assembly Buffer (GAB)), with a high concentration of nucleotide $(1 \mathrm{mM})$ is able to assemble into microtubules that contain nucleotide in the E-site (Table 2), (In the absence of nucleotide, the empty adduct is unable to assemble, and addition of $1 \mathrm{mM} \mathrm{GTP}$ after the 30 -min incubation at $37^{\circ} \mathrm{C}$ does not restore the ability to assemble, suggesting that the empty adduct is unstable at $37^{\circ} \mathrm{C}$ and gets denatured).

The fact that the passage through a G-25 column in a buffer at low nucleotide concentration removes the nucleotide bound to the zampanolide-tubulin adduct but not the nucleotide bound to the unmodified tubulin, indicates a decrease in the binding affinity of the protein for the nucleotide after modification. To confirm this hypothesis the binding affinity of tubulin and the zampanolide-adduct for a fluorescent bona fide analog of GTP (mant-GTP) was measured (Figure 6). The formation of the adduct significantly decreased the binding affinity at $25^{\circ} \mathrm{C}$ of tubulin for nucleotide from $12 \pm 2 \times 10^{5} \mathrm{M}^{-1}$ to $1.4 \pm 0.3 \times 10^{5} \mathrm{M}^{-1}$ in the case of the apotubulin-zampanolide adduct. 


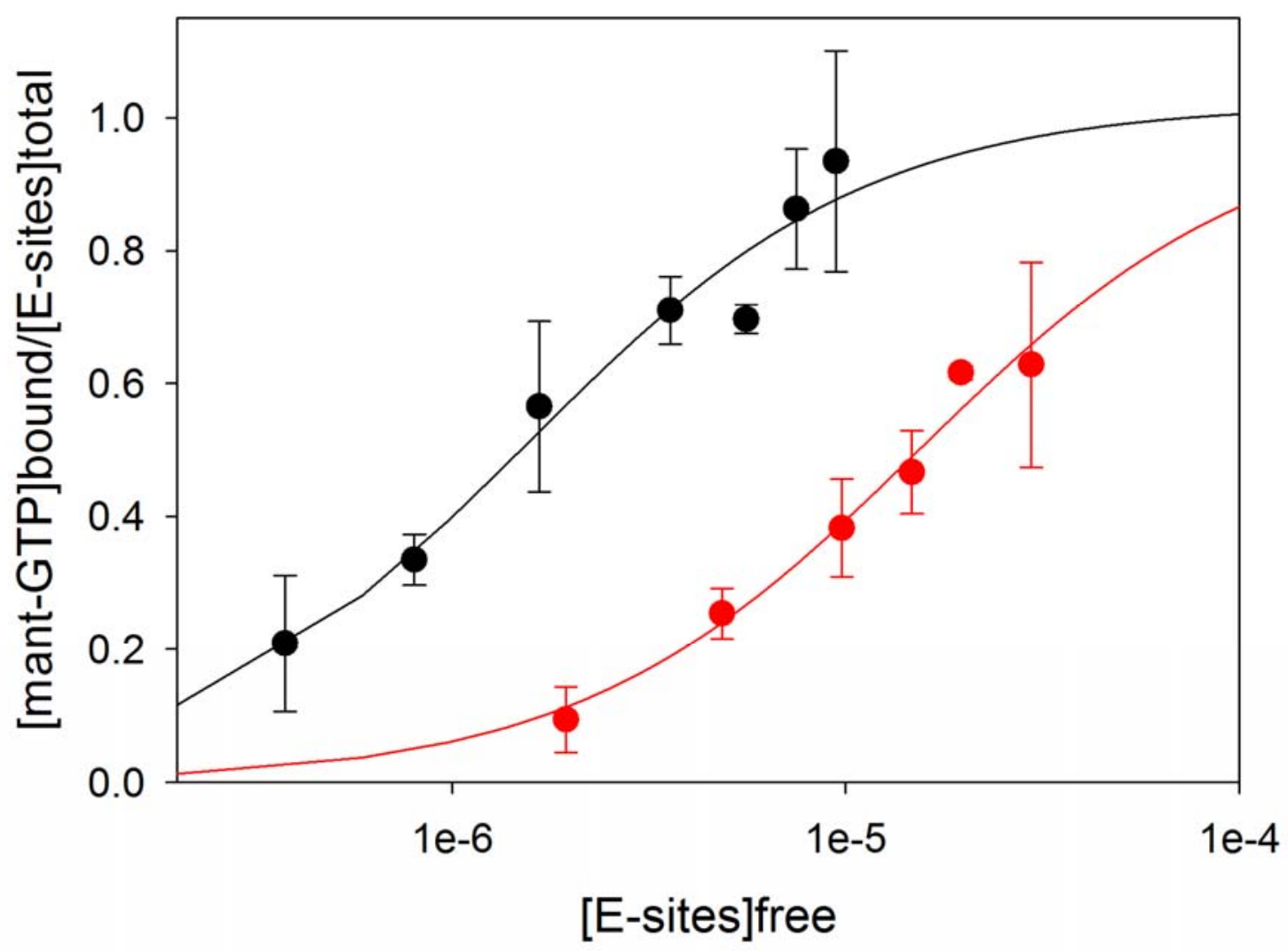

Figure 6.- Effect of adduct formation on the binding affinity at $25^{\circ} \mathrm{C}$ of tubulin for mantGTP. Titrations of mant-GTP with apo-tubulin (black circles and line) and apo-tubulinzampanolide adduct (red circles and line). Error bars are standard errors of three independent measurements.

Biochemical effects of tubulin activation by zampanolide: $\mathrm{Mg}^{2+}$ ions are required for the assembly and stability of microtubules. $\mathrm{Mg}^{2+}$ also has a significant influence on the interaction of the nucleotide with tubulin ${ }^{32}$. Similar to unligated tubulin, the zampanolide-adduct also requires $\mathrm{Mg}^{2+}$ for assembly and as shown in Figure 1, the formation of the adduct has no notable effect on the $\mathrm{Mg}^{2+}$-induced longitudinal tubulin-tubulin interactions responsible for the early aggregation events, thus indirectly suggesting that the assembly-promoting effect of zampanolide is exerted through the facilitation of lateral contacts. This would be in agreement with the idea that the 
zampanolide-adduct is a preorganized tubulin dimer that has its M-loop in the proper conformation for stable microtubule assembly, as suggested by the crystallographic data for the zampanolide-adduct ${ }^{17}$. In contrast, the M-loop of the unligated dimer is largely disorganized. Thus, it is plausible that the first step in lateral aggregation during nucleation from the tetramer to the oligomer state of tubulin could be more straightforward for the adduct than for unligatedtubulin, consistent with the crystallographic results in $\operatorname{ref}^{17}$.

Zampanolide binds at the luminal taxoid site in the tubulin dimer, and when binding to microtubules, it is likely to first interact with the pore type I site ${ }^{33}$ prior to translocation into the lumen. Binding at one site is considered to preclude binding at the other, since the stoichiometry of taxoid ligand binding to microtubules is $1: 1$, and an important $\beta$-tubulin loop (H6-H7) is part of both binding sites ${ }^{34,35}$. Given that zampanolide interacts with tubulin covalently, we have been able to directly confirm the mutually exclusive binding to the taxoid and pore type 1 site. Thus, binding of zampanolide completely abolishes binding to the pore type I site, since zampanolidesaturated microtubules cannot bind cyclostreptin or docetaxel even after extensive incubation with these ligands (data not shown). Binding at the taxoid site and the laulimalide/peloruside site, however, can occur simultaneously ${ }^{19}$, and formation of the zampanolide-adduct does not change the affinity of the laulimalide/peloruside site for peloruside binding. This implies that the synergy observed between peloruside and taxoid site binders for the inhibition of cancer cell proliferation in vitro does not occur at a structural, allosteric level, since compounds binding to either site affect the structuring of the same key M-loop ${ }^{19}$. This conclusion is concordant with the absence of an effect of laulimalide or peloruside on the binding of Flutax $-2^{36,37}$. Instead, the reported synergistic effects ${ }^{38,39}$ must be mediated by a different mechanism. For the taxoid site, nanomolar cytotoxic effects in cells are produced at ligand concentrations at which only about 2- 
$3 \%$ of the binding sites are occupied ${ }^{16}$, thus making it very unlikely that an individual tubulin molecule would have ligands bound to both the taxoid as well as the laulimalide/peloruside site in the absence of an allosterically-induced conformational change that would affect the binding constant of ligands for the second site. Instead, it is highly likely that a single ligated tubulin dimer has either its laulimalide/peloruside site or its taxoid site occupied. The bound molecules should therefore be scattered along a single microtubule, and the differential structural effects, M-loop re-structuring for the taxane site and M-loop re-structuring with matchmaking in the adjacent subunit ${ }^{19}$, could perturb microtubules in different ways. It is conceivable that these perturbations are super-additive, such that a microtubule containing dimers with $1 \%$ peloruside bound and $1 \%$ docetaxel bound may be more stable or its dynamics more suppressed than a microtubule with $2 \%$ docetaxel bound dimers. This would be reflected in synergistic effects on the inhibition of cell proliferation. In the present study we have demonstrated that there was no change in affinity for the colchicine binding site in the zampanolide-tubulin adduct. Although MTC showed significantly higher fluorescence when bound to the zampanolide-adduct compared to the unligated dimer, there was no difference in binding affinity of this ligand to the colchicine site. Based on crystallographic studies we can propose that the increase in the fluorescence of MTC in the presence of zampanolide in the paclitaxel site is due to a signal transmission from the paclitaxel site to the colchicine site through the $\mathrm{H} 7$ helix. Although no major conformational changes are described between the MTC-liganded tubulin and the binary MTC-zampanolide complex (no changes at all in the distances between the E-site nucleotide and the drug are observed), a re-orientation of the methylene group of $\beta \mathrm{A} 250$ is observed in the binary complex. This minor change affects the direct interaction with the trimethoxyphenyl ring 
of MTC and likely explains the observed increase in MTC-fluorescence. Such effects of taxoid site ligands on the structure of the colchicine site have not previously been described.

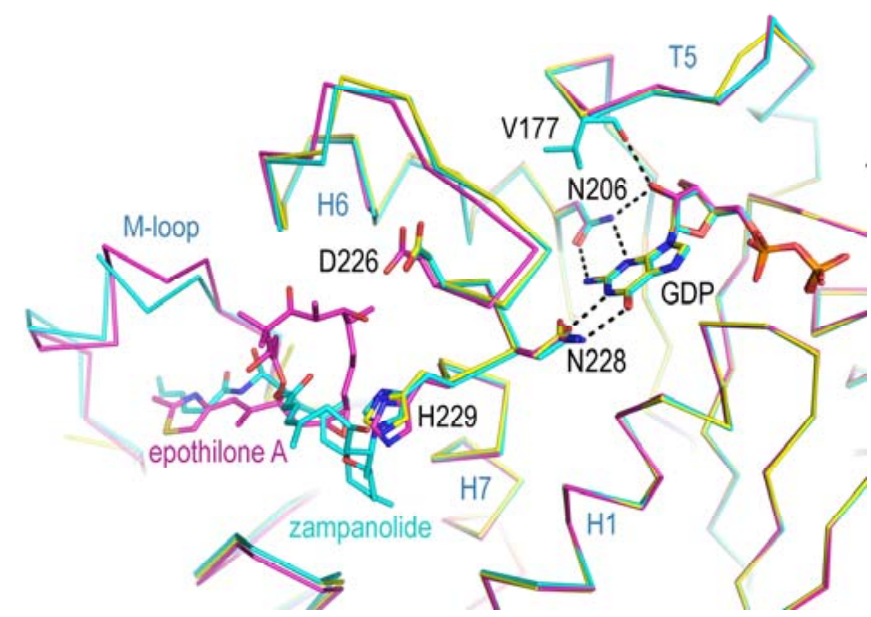

Figure 7.- Insight into the exchangeable nucleotide binding site of tubulin. Ribbon representation of the superimposed structures of $\beta$ tubulin in the absence of MSA $\left(\mathrm{T}_{2} \mathrm{R}\right.$-TTL-apo; PDB code: 4I55; yellow), in the presence of zampanolide $\left(\mathrm{T}_{2} \mathrm{R}\right.$-TTL-zampanolide; PDB code: 4I4T; cyan), and in the presence of epothilone $\mathrm{A}$ $\left(\mathrm{T}_{2} \mathrm{R}\right.$-TTL-epothilone $\mathrm{A}$; PDB code: 4I50; pink). Critical residues for ligand and nucleotide binding are in stick representation and are labeled. The secondary structure elements are labeled in blue. Hydrogen bonds are represented as dashed black lines.

The most significant effect observed upon formation of the zampanolide-adduct was loss of one order of magnitude of binding affinity for nucleotides at the E-site, indicating influence of the paclitaxel site on the Esite located in the longitudinal interface.

Electron microscopy studies ${ }^{21}$ of paclitaxel and nucleotide-stabilized microtubules have indicated that nucleotide hydrolysis leads to a compaction around the E-site at longitudinal interfaces that is inhibited in the presence of paclitaxel, thus suggesting that occupancy of the taxane site directly influences the E-site. Thus, occupation of the taxoid site by its ligand is able to mimic the effect of bound GTP, when the site is in fact occupied by GDP. The results presented in this work support the electron microscopy data and indicate that this structural effect is biochemically translated into changes of nucleotide binding to a tubulin molecule when the taxane site is occupied. It is difficult, however, to find the structural reason for this biochemical observation. To understand the structural effect of the covalent modification on the nucleotide 
binding site, we superimposed the crystal structures of apo-tubulin, and epothilone A- and zampanolide-bound tubulin (Figure 7). The overlay shows that the nucleotide is held in the binding site by five H-bonds formed with Asn206 (H6) and Asn228 (H7), and another H-bond between the ribose moiety of the nucleotide and the backbone carbonyl of Val177 (T5-loop). In addition, the purine ring is stacked between Cys12 and Tyr224. It would be logical to assume that the formation of the zampanolide adduct would modify at least one of these interactions, thus weakening the binding of the nucleotide. The most obvious candidates for this would be the two H-bonds formed with Asn228, since this residue is adjacent to His229, to which zampanolide is covalently attached in the adduct (The percentage of tubulin covalently modified at Asn228 by zampanolide is too insignificant to cause the stoichiometric effect observed).

However, the superposition of apo-tubulin with epothilone A-bound and zampanolidebound $T_{2} R$ complexes ${ }^{17}$ (Figure 7) indicate that, although there are significant differences in the orientation of the His229 side chain, the interactions of nucleotides with Asn228 are not disrupted in the zampanolide-adduct.

Instead, the superposition in Figure 7 suggests an indirect, allosteric effect on the E-site caused by a global effect of an MSA on tubulin structure, in a similar way as suggested by ${ }^{21}$. However, taking into account that the changes described by ${ }^{21}$ in the straight conformation of assembled tubulin may not be relevant to the unassembled, curved tubulin, we also compared the curved zampanolide-bound and the straight paclitaxel-bound structures by looking at the superposition of both the $\mathrm{N}$ - and $\mathrm{C}$-terminal domains of $\beta$-tubulin as well as the intermediate domains in a search for possible indirect structural effects related to dimer activation (Figure 8). The comparison of both conformational states indicates that the intermediate domain changes its relative orientation to both the amino- and carboxy-terminal domains, while the shape of the 


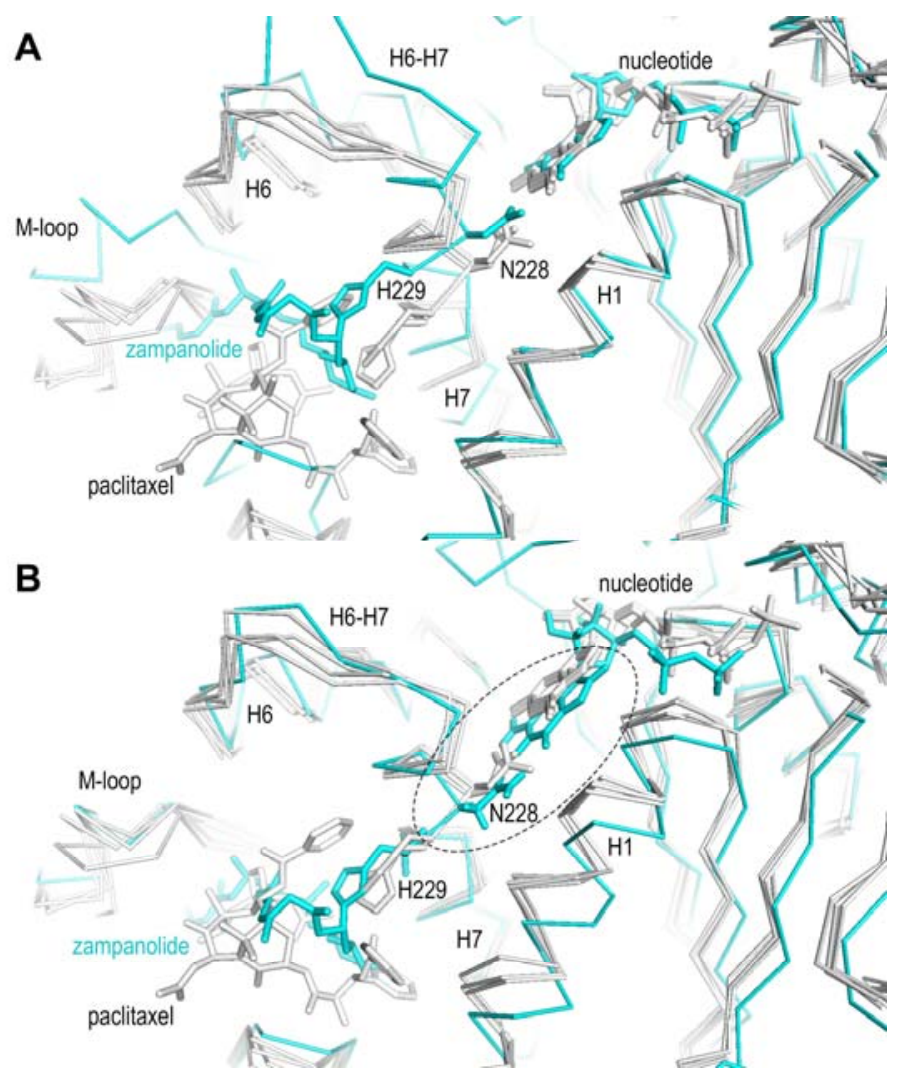

Figure 8.- Comparison between the curved zampanolide-bound and the straight conformation of tubulin. Ribbon representation of the superimposed structures of straight $\beta$-tubulin (PDB codes, 1JFF, 3J6E, $3 \mathrm{~J} 6 \mathrm{~F}$ and $3 \mathrm{~J} 6 \mathrm{G}$, grey) and the zampanolide-bound curved tubulin (PDB code: 4I4T; cyan) onto (A) the amino- and the carboxy-terminal domains, and (B) the intermediate domains. Critical residues and secondary structure elements are labeled. The key interaction between Asn228 and the nucleotide, which is perturbed by the zampanolide binding, is highlighted with a dashed black circle. taxane pocket remains unaltered. H6, the $\mathrm{H} 6-\mathrm{H} 7$ loop and $\mathrm{H} 7$ are the secondary structure elements that separate the nucleotide binding site from the taxane site. During the curved to straight transition the maintenance of the interactions of the nucleotide to Asn206 and Asn228 requires dynamic adaptation of these secondary structure elements. In the absence of a covalently bound ligand to His229 in the taxane site, Asn228 is free to move to maintain the coordination to the bound nucleotide. In contrast, the presence of the covalently bound zampanolide $^{10} \quad$ reduces this dynamicity, thereby preventing Asn228 from moving towards the

nucleotide (Figure 8). Moreover, binding of zampanolide to tubulin, as mentioned above, induces structuring of the M-loop ${ }^{17}$. If we compare all curved tubulin crystal forms, we find that the Mloop adopts different conformations in each form. Structuring of the M-loop requires the M-loop to move across the space covering the taxane site, flanking H6, to reach the conformation observed in the zampanolide structure. This transition may perturb H6 dynamics, thereby 
perturbing the nucleotide binding through Asn206. Although the crystallographic structures provide no information about the dynamic events that occur at the taxane/H7/E-site, it is reasonable to propose that a covalent binding agent such as zampanolide more strongly restricts these dynamic events compared to noncovalent binding ligands, since it fills the space between H7, the M-loop and H6 and allows transmission of movements between them. Helix H6 is also in direct contact with the T5 loop, one of the loops involved in the E-site compaction $^{21}$, which forms an H-bond with the ribose of the nucleotide. It is then likely that this H-bond, between the nucleotide and the backbone carbonyl of Val177, is perturbed upon zampanolide binding, resulting in loss of affinity for the nucleotide. Again, this shows communication or cross-talk between different binding sites in the tubulin molecule.

Understanding the mechanisms of activation of tubulin to cause polymer formation is essential for designing novel compounds with the capacity to modulate this activation. The use of zampanolide, a taxoid binding ligand that can bind covalently to His229, has allowed the study of a stable, activated tubulin dimer and revealed that the main changes in its properties are its superior ability to associate laterally and form microtubules and a 10 -fold decrease in the binding affinity for nucleotides. These findings are consistent with the presence of a prestructured M-loop, as found in the crystal structure of zampanolide-ligated tubulin ${ }^{17}$, as well as changes in the longitudinal interface of polymerized tubulin ${ }^{21}$. The perturbation of both the nucleotide binding site (which results in a loss of affinity of the nucleotide) and the colchicine site indicates communication between these sites and the taxane site, mediated by the M-loop restructuring. Given the fact that microtubule assembly fixes the exchangeable nucleotide in the binding site, which perturbs the taxane site, the assembly event may additionally contribute to the observed differences in binding affinity of MSAs for unassembled and assembled tubulin. 


\section{EXPERIMENTAL SECTION}

Proteins and ligands: Purified calf-brain tubulin ${ }^{40}$, stabilized, crosslinked microtubules $^{34,41}$, and recombinant human protein containing a stathmin-like binding domain $(\mathrm{RB} 3-\mathrm{SLD})^{42}$ were prepared as previously described. The tubulin-zampanolide adduct was prepared by a 30 min incubation of tubulin with a $10 \%$ molar excess of zampanolide in the cold, and its formation was checked by mass spectrometry as described ${ }^{10}$.

Natural zampanolide was isolated and purified from the marine sponge Cacospongia mycofijiensis collected from 'Eua and Vava'u, Tonga as described ${ }^{13}$. Peloruside A was isolated and purified from the marine sponge Mycale hentscheli collected from Pelorus Sound, New Zealand as described ${ }^{43}$. Synthetic zampanolide was prepared as described ${ }^{14,44}$ and was identical in all respects with the natural material. Aliquots of the compounds were dried to constant weight and dissolved in spectroscopic DMSO (Merck, Darmstadt, Germany). Spectroscopic analysis of the compounds was performed as described ${ }^{10}$. Docetaxel (Taxotere $\left.{ }^{\circledR}\right)$ was kindly provided by Rhône Poulenc Rorer, Aventis (Schiltigheim, France). The colchicine analog 2-methoxy-5(2,3,4-trimethoxyphenyl)-2,4,6-cyclo heptatrien-1-one (MTC) was a kind gift from Dr. T. Fitzgerald (Florida A\&M University, FL, USA). Mant-GTP (a bona fide fluorescent analog of GTP) was from Jena Biosciences (Jena, Germany).

Analytical ultracentrifugation: Sedimentation velocity experiments were performed to determine the effect of adduct formation on the ability of tubulin to oligomerize under different conditions. Tubulin was equilibrated in PEDTA buffer (10 mM NaPi (sodium phosphate), $1 \mathrm{mM}$ EDTA, $\mathrm{pH}$ 7.0) and then diluted to the desired concentration. Zampanolide, epothilone B, or DMSO (vehicle) was added to the samples at the desired concentration in addition to GTP or 
GDP, $\mathrm{MgCl}_{2}$, and DTT. The stathmin-like domain RB3-SLD was added depending on the experimental conditions desired. The $\mathrm{Mg}^{2+}$ free concentrations were calculated by correcting for the cation binding to phosphate, nucleotide and EDTA as described ${ }^{45}$.

Samples were incubated at $25^{\circ} \mathrm{C}$ for $30 \mathrm{~min}$ and centrifuged at $40,000 \mathrm{rpm}$ using an An50Ti eight-hole rotor with double-sector Epon-charcoal centrepieces in a Beckman Optima XLI analytical ultracentrifuge with absorption and interference optics. Differential sedimentation coefficient distributions $\mathrm{c}(\mathrm{s})$ were calculated by least-squares boundary modeling of the experimental data using the program SEDFIT ${ }^{46}$ and corrected to water and $20^{\circ} \mathrm{C}$.

Effect of zampanolide tubulin-adduct formation on MSA and colchicine binding sites: The effect of adduct formation on the affinity of the laulimalide/peloruside site was measured using HPLC analysis by monitoring the binding of ligands to stabilized taxoid binding sites. Stabilized crosslinked microtubules $(0.6 \mu \mathrm{M})$ were incubated with a $10 \%$ stoichiometric excess of zampanolide or the equivalent volume of DMSO for $30 \mathrm{~min}$ at $25^{\circ} \mathrm{C}$ in $3.4 \mathrm{M}$ glycerol, 10 mM sodium phosphate, $1 \mathrm{mM}$ EDTA, $6 \mathrm{mM} \mathrm{MgCl} 2,0.1 \mathrm{mM}$ GTP, pH 6.7 (GAB buffer) plus. Peloruside (0.1-10 $\mu \mathrm{M})$ or an equivalent volume of DMSO was then added to the samples, followed by a further incubation for $30 \mathrm{~min}$ at $25^{\circ} \mathrm{C}$. The samples were then centrifuged for 20 min at $25^{\circ} \mathrm{C}$ at $50,000 \mathrm{rpm}$ in a TLA 120.2 rotor in an Optima TLX centrifuge (Beckman, Palo Alto, CA) to separate out the supernatants and pellets. The pellets were resuspended in $10 \mathrm{mM}$ $\mathrm{NaPi}$. An internal standard of $10 \mu \mathrm{M}$ docetaxel was added to all samples and the compounds extracted three times with one reaction volume of $\mathrm{CH}_{2} \mathrm{Cl}_{2}$. The extract was dried and the sample resuspended in $35 \mu \mathrm{L} \mathrm{v} / \mathrm{v} 55 \%$ methanol in water. The compounds were analyzed using an Agilent 1100 Series instrument employing a Supercosil, LC18 DB HPLC column of dimensions 
$250 \times 4.6 \mathrm{~mm}$ and a bead diameter of $5 \mathrm{~mm}$. The column was developed with a gradient of 13 $\min 55 \%$ methanol in water, $10 \mathrm{~min} 70 \%$ methanol in water, and $10 \mathrm{~min} 55 \%$ methanol in water at a flow rate of $1 \mathrm{~mL} / \mathrm{min}$. The absorbance was followed at 205 and $230 \mathrm{~nm}$. The binding constant of peloruside was calculated employing EQUIGRA V5.0 ${ }^{47}$ and compared between unligated and zampanolide-ligated tubulin.

The effect of adduct formation on the colchicine site was studied using MTC, a synthetic bicyclic derivative of colchicine that binds quickly and reversibly to the colchicine site on tubulin $^{48}$, and upon binding emits fluorescence at $425 \mathrm{~nm}^{49}$. Tubulin was prepared in $10 \mathrm{mM}$ NaPi, $0.1 \mathrm{mM}$ GTP, $\mathrm{pH} 7.0$ buffer as described above, and incubated with a $10 \%$ stoichiometric excess of zampanolide or an equivalent volume of DMSO for $1.5 \mathrm{hr}$ at $4{ }^{\circ} \mathrm{C}$. The fluorescence spectrum of MTC was measured using a FluoroMax-2® ISA ${ }^{\circledR}$ Jobin Yvon-Spex spectrofluorometer $\left(\lambda_{\text {exc }} 350 \mathrm{~nm}, \lambda_{\text {ems }} 425 \mathrm{~nm}\right)$. The fluorescence spectrum of $10 \mu \mathrm{M}$ tubulin or $10 \mu \mathrm{M}$ zampanolide-ligated tubulin in PEDTA buffer with increasing concentrations of MTC (0 $\mu \mathrm{M}-50 \mu \mathrm{M})$ was measured as above.

Effect of zampanolide binding on the exchangeable nucleotide site: Tubulin was equilibrated in PEDTA buffer as described. To measure the nucleotide content of dimeric tubulin, $40 \mu \mathrm{M}$ tubulin equilibrated in PEDTA buffer was incubated with $10 \%$ excess ligand or an equivalent volume of DMSO for $1.5 \mathrm{hr}$ at $25^{\circ} \mathrm{C}$. The unbound nucleotide from the samples was removed by passing the solution through a Sephadex G-25 column $(25 \mathrm{x} 0.9 \mathrm{~cm})$ (GE Healthcare Bioscience, Upsala, Sweden), pre-equilibrated in PEDTA buffer plus $10 \mu \mathrm{M}$ GTP, $1.5 \mu \mathrm{M} \mathrm{MgCl}_{2}$ at $4^{\circ} \mathrm{C}$. A $50 \mu \mathrm{L}$ aliquot of sample was diluted 20 -fold in $10 \mathrm{mM} \mathrm{NaPi}, 1 \% \mathrm{SDS}$ 
$\mathrm{pH} 7.0$, and the concentration of tubulin and nucleotide in each sample was quantified as described $^{45}$.

To measure the nucleotide in GAB buffer-assembled microtubules, tubulin and adduct prepared as described above was supplemented with $3.4 \mathrm{M}$ glycerol, $1 \mathrm{mM}$ EGTA, $\mathrm{MgCl}_{2}$ up to $6 \mathrm{mM}$ and no nucleotide or $1 \mathrm{mM} \mathrm{GTP}$ and assembled at $37^{\circ} \mathrm{C}$ for $30 \mathrm{~min}$. The samples were then centrifuged at $50,000 \mathrm{rpm}$ at $37^{\circ} \mathrm{C}$ for $20 \mathrm{~min}$ and the pellets washed in three volumes of $500 \mu \mathrm{L}$ warm GAB buffer without GTP. The pellets were dissolved in $10 \mathrm{mM} \mathrm{NaPi}$ and the concentration of tubulin measured as above. Nucleotide was quantified as described ${ }^{45}$. Small aliquots of the assembly solutions were routinely adsorbed to carbon-coated Formvar films on 400-mesh cooper grids (for a time in minutes that was roughly equal to the reciprocal of the tubulin concentration in $\mathrm{mg} / \mathrm{mL}$ ), stained for $1 \mathrm{~min}$ with $2 \%$ uranyl acetate, and observed in a JEOL JEM-1230 electron microscope.

Nucleotide extraction and HPLC separation were then carried out on all the above samples to determine the concentration of each nucleotide, as described ${ }^{50}$, with modifications as described in $^{45}$.

The binding constants of mant-GTP to the tubulin dimer and the adduct were measured employing an anisotropy fluorescence assay as described ${ }^{51}$. The anisotropy of a $2 \mu \mathrm{M}$ solution of mant-GTP in $10 \mathrm{mM} \mathrm{NaPi}, 1.5 \mathrm{mM} \mathrm{MgCl} 2 \mathrm{pH} 7.0$ was measured as described ${ }^{51}$ in the presence of growing amounts of apo-tubulin or apo-tubulin-zampanolide adduct (depleted from the E-site nucleotide with active charcoal as described ${ }^{52}$ ). The fraction of intact E-site binding sites in apotubulin $(0.29 \pm 0.04)$ and apo-tubulin-zampanolide adduct $(0.30 \pm 0.02)$ after the active charcoal treatment was determined by measuring the recovery of GTP-binding of the treated protein. The content of nucleotide extracted after the treatment with active charcoal was found to be 
$0.35 \pm 0.01 \mathrm{~mol}$ of nucleotide per dimer of tubulin for the case of the apo-tubulin and $0.49 \pm 0.01$ for the case of apo-tubulin-zampanolide. This nucleotide was considered bound to the N-site. Then, $50 \mu \mathrm{M}$ apo-tubulin and apo-tubulin-zampanolide adduct were incubated with $1 \mathrm{mM}$ GTP in $10 \mathrm{mM} \mathrm{NaPi}, 1.5 \mathrm{mM} \mathrm{MgCl} 2 \mathrm{pH} 7.0$ for $30 \mathrm{~min}$ and the unbound nucleotide removed with a pass through a Sephadex G-25 column $(20 \mathrm{x} 0.9 \mathrm{~cm})$ equilibrated in $100 \mu \mathrm{M}$ GTP in $10 \mathrm{mM} \mathrm{NaPi}$, $1.5 \mathrm{mM} \mathrm{MgCl} 2 \mathrm{pH}$ 7.0. The extracted nucleotide content was found to be $0.64 \pm 0.03 \mathrm{~mol}$ of nucleotide per mol of tubulin for the apo-tubulin and $0.79 \pm 0.01$ for the apo-tubulin-zampanolide adduct. The differences in the two values was considered to be the fraction of active E-sites for each protein.

The values of the anisotropy measured were analyzed as described in ${ }^{51}$ to calculate the binding constants of the fluorescent nucleotide to apo-tubulin and apo-tubulin-zampanolide.

Crystallization, data collection and structure determination: Crystals of $\mathrm{T}_{2} \mathrm{R}$-TTL were grown as described by Prota et al. ${ }^{17,29}$. Briefly, the T2R-TTL complex was crystallized by the vapor-diffusion method at $20^{\circ} \mathrm{C}$. Crystals grew over night in precipitant solution consisting of 5\% PEG 4K, 12-14\% glycerol, $30 \mathrm{mM} \mathrm{MgCl} 2,30 \mathrm{mM} \mathrm{CaCl}_{2}, 100 \mathrm{mM}$ MES/Imidazole, $\mathrm{pH}$ 6.7. $\mathrm{T}_{2} \mathrm{R}$-TTL-MTC and $\mathrm{T}_{2} \mathrm{R}$-TTL-MTC-zampanolide complexes were prepared by overnight soaking of crystals at $20^{\circ} \mathrm{C}$ in reservoir solutions containing either $1 \mathrm{mM} \mathrm{MTC}$ or a mixture of both $10 \mathrm{mM}$ MTC and $5 \mathrm{mM}$ zampanolide, 10\% PEG 4K and 13-16\% glycerol, followed by a consecutive transfer to reservoir solutions containing $15 \%$ (only $\mathrm{T}_{2} \mathrm{R}-\mathrm{TTL}-\mathrm{MTC}$ ) and $20 \%$ glycerol before flash-cooling in liquid nitrogen. Native data were collected at $100 \mathrm{~K}$ at beamlines X06SA ( $\left.\mathrm{T}_{2} \mathrm{R}-\mathrm{TTL}-\mathrm{MTC}\right)$ and X06DA ( $\mathrm{T}_{2} \mathrm{R}$-TTL-MTC-zampanolide) of the Swiss Light Source (Paul Scherrer Institut, Villigen, Switzerland). Data were processed and merged with $\mathrm{XDS}^{53}$. The 
structures were determined by the difference Fourier method using the phases of the $\mathrm{T}_{2} \mathrm{R}$-TTL complex (PDB ID 4I4T) in the absence of ligands and solvent molecules as a starting point for refinement. The models were first fitted by several cycles of rigid body refinement followed by simulated annealing and restrained refinement in Phenix ${ }^{54}$. The resulting models were further improved through iterative model rebuilding in $\operatorname{Coot}^{55}$ and refinement in Phenix. The quality of the structures was assessed with MolProbity ${ }^{56}$. Data collection and refinement statistics are given in Table 1.

Chains in the $\mathrm{T}_{2} \mathrm{R}$-TTL complex were defined as follows: chain A, $\alpha 1$-tubulin; chain $\mathrm{B}, \beta 1$ tubulin; chain C, $\alpha 2$-tubulin; chain D, $\beta 2$-tubulin; chain E, RB3; chain F, TTL (Figure 4). Structure visualization, molecular editing and figure preparation were performed with the PyMOL molecular graphics system ${ }^{57}$.

The atomic coordinates and structure factors have been deposited in the Protein Data Bank (www.rcsb.org) under accession codes 5NFZ (T2R-TTL-MTC) and 5NG1 (T2R-TTL-MTCzampanolide).

Molecular modeling: The superpositions shown in Figures 7 and 8 were prepared using the align-function in PyMOL ${ }^{57}$. For Figure 7, both the D-chains of the amino- (residues 1-205) and the carboxy-terminal (residues 385-441) domains of the $\mathrm{T}_{2} \mathrm{R}$-TTL-apo (PDB code: 4I55; rmsd=0.218 $\AA, 233 \mathrm{C}_{\alpha}$ ) and the $\mathrm{T}_{2} \mathrm{R}$-TTL-epothilone A (PDB code: $4 \mathrm{I} 50$; rmsd=0.218 $\AA, 233$ $\mathrm{C}_{\alpha}$ ) complexes were superimposed onto the corresponding domains of T2R-TTL-zampanolide (PDB code: 4I4T). For Figure 8, the D-chain of $\mathrm{T}_{2} \mathrm{R}$-TTL-zampanolide (PDB code: 4I4T) was superimposed onto both the amino and the carboxy-terminal, and the intermediate $\beta$-tubulin domains (residues 206-384) of GDP-microtubules (PDB code: 3J6F; rmsd (N-/C-)=0.986 $\AA, 233$ 
$\mathrm{C}_{\alpha} ;$ rmsd (inter)=1.021 $\AA, 145 \mathrm{C}_{\alpha}$ ), GMPCPP-microtubules (PDB code: $3 \mathrm{~J} 6 \mathrm{E} ; \mathrm{rmsd}(\mathrm{N}-/ \mathrm{C}-)=$ $0.815 \AA, 230 \mathrm{C}_{\alpha}$; rmsd (inter) $=0.946 \AA, 141 \mathrm{C}_{\alpha}$ ) and paclitaxel-stabilized GDP-microtubules (PDB code: 3J6G; rmsd (N-/C-)=0.934 $\AA, 240 \mathrm{C}_{\alpha}$; rmsd (inter)=1.193 $\AA, 156 \mathrm{C}_{\alpha}$ ), and the electron crystallography structure of $\beta$-tubulin (PDB code: $1 \mathrm{JFF}$; rmsd (N-/C-)=0.917 $\AA, 232 \mathrm{C}_{\alpha}$; $\operatorname{rmsd}\left(\right.$ inter) $\left.=1.192 \AA, 147 \mathrm{C}_{\alpha}\right)$.

\section{ACKNOWLEDGEMENTS}

We thank Rhône Poulenc Rorer Aventis for docetaxel, Peter T. Northcote for peloruside, and Ganadería Fernando Díaz for calf brains for tubulin purification; C. Alfonso and J. Luque for assistance and analysis of analytical ultracentrifugation experiments. J.F. received a short-term fellowship from EMBO. B.P. acknowledges Comunidad Autonoma de Madrid and Fondo Social Europeo for "CONTRATO DE APOYO A LA INVESTIGACIÓN". This work was supported in part by grants BFU2016-75319-R (AEI/FEDER, UE) from Ministerio de Economia y Competitividad and the Cancer Society of New Zealand, and the Wellington Medical Research Foundation (JJF \& JHM). The authors acknowledge networking contribution by the COST Action CM1407 "Challenging organic syntheses inspired by nature - from natural products chemistry to drug discovery". The CNIC is supported by the Ministerio de Ciencia e Innovación and the Fundación Pro CNIC.

Notes: The authors declare no competing financial interest. 


\section{References}

1. Jordan, M. A.; Wilson, L., Microtubules as a target for anticancer drugs. Nat Rev Cancer 2004, 4, (4), 253-65.

2. Field, J.; Kanakkanthara, A.; Miller, J., Microtubule-targeting agents are clinically successful due to both mitotic and interphase impairment of microtubule function. Bioorganic \& Medicinal Chemistry 2014, 22, (18), 5050-5059.

3. Komlodi Pasztor, E.; Sackett, D.; Wilkerson, J.; Fojo, T., Mitosis is not a key target of microtubule agents in patient tumors. Nature Reviews Clinical Oncology 2011, 8, (4), 244-250.

4. Gainor, J. F.; Shaw, A. T., Emerging Paradigms in the Development of Resistance to Tyrosine Kinase Inhibitors in Lung Cancer. Journal of Clinical Oncology 2013, 31, (31), 39873996.

5. Gottesman, M.; Fojo, T.; Bates, S., Multidrug resistance in cancer: Role of ATPdependent transporters. Nature Reviews. Cancer 2002, 2, (1), 48-58.

6. Kavallaris, M., Microtubules and resistance to tubulin-binding agents. Nature Reviews Cancer 2010, 10, (3), 194-204.

7. Dumontet, C.; Jordan, M. A., Microtubule-binding agents: a dynamic field of cancer therapeutics. Nat Rev Drug Discov 2010, 9, (10), 790-803.

8. Sackett, D., Antimicrotubule agents that bind covalently to tubulin. In The Role of Microtubules in Cell Biology, Neurobiology, and Oncology, Fojo, A., Ed. Humana Press: 2008; pp 281-306.

9. Buey, R. M.; Calvo, E.; Barasoain, I.; Pineda, O.; Edler, M. C.; Matesanz, R.; Cerezo, G.; Vanderwal, C. D.; Day, B. W.; Sorensen, E. J.; Lopez, J. A.; Andreu, J. M.; Hamel, E.; Díaz, J. 
F., Cyclostreptin binds covalently to microtubule pores and lumenal taxoid binding sites. Nature Chem. Biol. 2007, 3, (2), 117-25.

10. Field, J. J.; Pera, B.; Calvo, E.; Canales, A.; Zurwerra, D.; Trigili, C.; RodriguezSalarichs, J.; Matesanz, R.; Kanakkanthara, A.; Wakefield, S. J.; Singh, A. J.; Jimenez-Barbero, J.; Northcote, P.; Miller, J. H.; Lopez, J. A.; Hamel, E.; Barasoain, I.; Altmann, K. H.; Diaz, J. F., Zampanolide, a potent new microtubule-stabilizing agent, covalently reacts with the taxane luminal site in tubulin alpha,beta-heterodimers and microtubules. Chem Biol 2012, 19, (6), 68698.

11. Risinger, A. L.; Li, J.; Bennett, M. J.; Rohena, C. C.; Peng, J.; Schriemer, D. C.; Mooberry, S. L., Taccalonolide binding to tubulin imparts microtubule stability and potent in vivo activity. Cancer Res 2013, 73, (22), 6780-92.

12. Singh, J.; Petter, R.; Baillie, T.; Whitty, A., The resurgence of covalent drugs. Nature reviews. Drug discovery 2011, 10, (4), 307-317.

13. Field, J. J.; Singh, A. J.; Kanakkanthara, A.; Halafihi, T.; Northcote, P. T.; Miller, J. H., Microtubule-Stabilizing Activity of Zampanolide, a Potent Macrolide Isolated from the Tongan Marine Sponge Cacospongia mycofijiensis. Journal of Medicinal Chemistry 2009, 52, (22), 7328-7332.

14. Zurwerra, D.; Gertsch, J.; Altmann, K. H., Synthesis of (-)-Dactylolide and 13Desmethylene-(-)-dactylolide and Their Effects on Tubulin. Organic Letters 2010, 12, (10), $2302-2305$.

15. Lowe, J.; Li, H.; Downing, K. H.; Nogales, E., Refined structure of alpha beta-tubulin at 3.5 Å resolution. Journal of molecular biology 2001, 313, (5), 1045-57. 
16. Matesanz, R.; Barasoain, I.; Yang, C.; Wang, L.; Li, X.; De Ines, C.; Coderch, C.; Gago, F.; Jiménez-Barbero, J.; Andreu, J. M.; Fang, W.; Díaz, J. F., Optimization of taxane binding to microtubules. Binding affinity decomposition and incremental construction of a high-affinity analogue of paclitaxel. Chem Biol 2008, 15, 573-585.

17. Prota, A. E.; Bargsten, K.; Zurwerra, D.; Field, J. J.; Diaz, J. F.; Altmann, K. H.; Steinmetz, M. O., Molecular mechanism of action of microtubule-stabilizing anticancer agents. Science 2013, 339, (6119), 587-90.

18. Nogales, E.; Whittaker, M.; Milligan, R. A.; Downing, K. H., High-resolution model of the microtubule. Cell 1999, 96, (1), 79-88.

19. Prota, A. E.; Bargsten, K.; Northcote, P. T.; Marsh, M.; Altmann, K. H.; Miller, J. H.; Diaz, J. F.; Steinmetz, M. O., Structural basis of microtubule stabilization by laulimalide and peloruside A. Angew Chem Int Ed Engl 2014, 53, (6), 1621-5.

20. Amos, L. A.; Lowe, J., How Taxol stabilises microtubule structure. Chem Biol 1999, 6, (3), R65-9.

21. Alushin, G. M.; Lander, G. C.; Kellogg, E. H.; Zhang, R.; Baker, D.; Nogales, E., HighResolution Microtubule Structures Reveal the Structural Transitions in alpha beta-Tubulin upon GTP Hydrolysis. Cell 2014, 157, (5), 1117-1129.

22. Kellogg, E. H.; Hejab, N. M. A.; Howes, S.; Northcote, P.; Miller, J. H.; Díaz, J. F.; Downing, K. H.; Nogales, E., Insights into the Distinct Mechanisms of Action of Taxane and Non-Taxane Microtubule Stabilizers from Cryo-EM Structures. Journal of Molecular Biology 2017, 429, (5), 633-646. 
23. Díaz, J. F.; Andreu, J. M.; Diakun, G.; Towns-Andrews, E.; Bordas, J., Structural intermediates in the assembly of taxoid-induced microtubules and GDP-tubulin double rings: time-resolved X-ray scattering. Biophys J 1996, 70, (5), 2408-20.

24. Frigon, R. P.; Timasheff, S. N., Magnesium-induced self-association of calf brain tubulin. I. Stoichiometry. Biochemistry 1975, 14, (21), 4559-66.

25. Díaz, J. F.; Pantos, E.; Bordas, J.; Andreu, J. M., Solution structure of GDP-tubulin double rings to $3 \mathrm{~nm}$ resolution and comparison with microtubules. $J$ Mol Biol 1994, 238, (2), 214-25.

26. Pera, B.; Razzak, M.; Trigili, C.; Pineda, O.; Canales, A.; Buey, R. M.; Jimenez-Barbero, J.; Northcote, P. T.; Paterson, I.; Barasoain, I.; Diaz, J. F., Molecular Recognition of Peloruside A by Microtubules. The C24 Primary Alcohol is Essential for Biological Activity. Chembiochem 2010, 11, (12), 1669-1678.

27. La Regina, G.; Edler, M. C.; Brancale, A.; Kandil, S.; Coluccia, A.; Piscitelli, F.; Hamel, E.; De Martino, G.; Matesanz, R.; Diaz, J. F.; Scovassi, A. I.; Prosperi, E.; Lavecchia, A.; Novellino, E.; Artico, M.; Silvestri, R., Arylthioindole inhibitors of tubulin polymerization. 3. Biological evaluation, structure-activity relationships and molecular modeling studies. $J$ Med Chem 2007, 50, (12), 2865-74.

28. Menendez, M.; Rivas, G.; Diaz, J. F.; Andreu, J. M., Control of the structural stability of the tubulin dimer by one high affinity bound magnesium ion at nucleotide N-site. J Biol Chem 1998, 273, (1), 167-176.

29. Prota, A. E.; Magiera, M. M.; Kuijpers, M.; Bargsten, K.; Frey, D.; Wieser, M.; Jaussi, R.; Hoogenraad, C. C.; Kammerer, R. A.; Janke, C.; Steinmetz, M. O., Structural basis of tubulin tyrosination by tubulin tyrosine ligase. J Cell Biol 2013, 200, (3), 259-70. 
30. Nogales, E.; Downing, K. H.; Amos, L. A.; Lowe, J., Tubulin and FtsZ form a distinct family of GTPases. Nat Struct Biol 1998, 5, (6), 451-8.

31. Weisenberg, R. C.; Borisy, G. G.; Taylor, E. W., The colchicine-binding protein of mammalian brain and its relation to microtubules. Biochemistry 1968, 7, (12), 4466-79.

32. Correia, J. J.; Baty, L. T.; Williams, R. C., Jr., Mg2+ dependence of guanine nucleotide binding to tubulin. J Biol Chem 1987, 262, (36), 17278-84.

33. Field, J. J.; Diaz, J. F.; Miller, J. H., The Binding Sites of Microtubule-Stabilizing Agents. Chemistry \& Biology 2013, 20, (3), 301-315.

34. Díaz, J. F.; Barasoain, I.; Andreu, J. M., Fast kinetics of Taxol binding to microtubules. Effects of solution variables and microtubule-associated proteins. J Biol Chem 2003, 278, (10), 8407-19.

35. Magnani, M.; Maccari, G.; Andreu, J. M.; Diaz, J. F.; Botta, M., Possible binding site for paclitaxel at microtubule pores. FEBS J 2009, 276, (10), 2701-12.

36. Pryor, D. E.; O'Brate, A.; Bilcer, G.; Díaz, J. F.; Wang, Y.; Kabaki, M.; Jung, M. K.; Andreu, J. M.; Ghosh, A. K.; Giannakakou, P.; Hamel, E., The microtubule stabilizing agent laulimalide does not bind in the taxoid site, kills cells resistant to paclitaxel and epothilones, and may not require its epoxide moiety for activity. Biochemistry 2002, 41, (29), 9109-15.

37. Gaitanos, T. N.; Buey, R. M.; Díaz, J. F.; Northcote, P. T.; Teesdale-Spittle, P.; Andreu, J. M.; Miller, J. H., Peloruside A does not bind to the taxoid site on beta-tubulin and retains its activity in multidrug-resistant cell lines. Cancer Res 2004, 64, (15), 5063-7.

38. Hamel, E.; Day, B. W.; Miller, J. H.; Jung, M. K.; Northcote, P. T.; Ghosh, A. K.; Curran, D. P.; Cushman, M.; Nicolaou, K. C.; Paterson, I.; Sorensen, E. J., Synergistic effects of 
peloruside A and laulimalide with taxoid site drugs, but not with each other, on tubulin assembly. Mol Pharmacol 2006, 70, (5), 1555-64.

39. Wilmes, A.; O'Sullivan, D.; Chan, A.; Chandrahasen, C.; Paterson, I.; Northcote, P.; La Flamme, A.; Miller, J.; Osullivan, D., Synergistic interactions between peloruside A and other microtubule-stabilizing and destabilizing agents in cultured human ovarian carcinoma cells and murine T cells. Cancer Chemotherapy and Pharmacology 2011, 68, (1), 117-126.

40. Andreu, J. M., Large Scale Purification of Brain Tubulin. In Methods in Molecular Medicine, Zhou, J., Ed. Humana Press Inc.: Totowa, NJ, 2007; Vol. 137, pp 17-28.

41. Díaz, J. F.; Strobe, R.; Engelborghs, Y.; Souto, A. A.; Andreu, J. M., Molecular recognition of taxol by microtubules. Kinetics and thermodynamics of binding of fluorescent taxol derivatives to an exposed site. J Biol Chem 2000, 275, (34), 26265-76.

42. Barbier, P.; Dorleans, A.; Devred, F.; Sanz, L.; Allegro, D.; Alfonso, C.; Knossow, M.; Peyrot, V.; Andreu, J. M., Stathmin and interfacial microtubule inhibitors recognize a naturally curved conformation of tubulin dimers. J Biol Chem 2010, 285, (41), 31672-81.

43. West, L. M.; Northcote, P. T.; Battershill, C. N., Peloruside A: A potent cytotoxic macrolide isolated from the New Zealand marine sponge Mycale sp. J Org Chem 2000, 65, (2), 445-449.

44. Zurwerra, D.; Glaus, F.; Betschart, L.; Schuster, J.; Gertsch, J.; Ganci, W.; Altmann, K.H., Total Synthesis of (-)-Zampanolide and Structure-Activity Relationship Studies on (-)Dactylolide Derivatives. Chemistry - A European Journal 2012, 18, (52), 16868-16883.

45. Díaz, J. F.; Andreu, J. M., Assembly of purified GDP-tubulin into microtubules induced by taxol and taxotere: reversibility, ligand stoichiometry, and competition. Biochemistry 1993, $32,(11), 2747-55$. 
46. Schuck, P., Size-distribution analysis of macromolecules by sedimentation velocity ultracentrifugation and lamm equation modeling. Biophys $J$ 2000, 78, (3), 1606-19.

47. Diaz, J. F.; Buey, R. M., Characterizing ligand-microtubule binding by competition methods. Methods Mol Med 2007, 137, 245-60.

48. Fitzgerald, T. J., Molecular features of colchicine associated with antimitotic activity and inhibition of tubulin polymerization. Biochem Pharmacol 1976, 25, (12), 1383-7.

49. Andreu, J. M.; Gorbunoff, M. J.; Lee, J. C.; Timasheff, S. N., Interaction of tubulin with bifunctional colchicine analogues: an equilibrium study. Biochemistry 1984, 23, (8), 1742-52.

50. Seckler, R.; Wu, G. M.; Timasheff, S. N., Interactions of tubulin with guanylyl-(betagamma-methylene)diphosphonate. Formation and assembly of a stoichiometric complex. $J$ Biol Chem 1990, 265, (13), 7655-61.

51. Schaffner-Barbero, C.; Gil-Redondo, R.; Ruiz-Avila, L. B.; Huecas, S.; Lappchen, T.; den Blaauwen, T.; Diaz, J. F.; Morreale, A.; Andreu, J. M., Insights into nucleotide recognition by cell division protein FtsZ from a mant-GTP competition assay and molecular dynamics. Biochemistry 2010, 49, (49), 10458-72.

52. Fishback, J. L.; Yarbrough, L. R., Interaction of 6-mercapto-GTP with bovine brain tubulin. Equilibrium aspects. J Biol Chem 1984, 259, (3), 1968-73.

53. Kabsch, W., XDS. Acta Crystallographica Section D 2010, 66, (2), 125-132.

54. Adams, P. D.; Afonine, P. V.; Bunkoczi, G.; Chen, V. B.; Davis, I. W.; Echols, N.; Headd, J. J.; Hung, L.-W.; Kapral, G. J.; Grosse-Kunstleve, R. W.; McCoy, A. J.; Moriarty, N. W.; Oeffner, R.; Read, R. J.; Richardson, D. C.; Richardson, J. S.; Terwilliger, T. C.; Zwart, P. H., PHENIX: a comprehensive Python-based system for macromolecular structure solution. Acta Crystallographica Section D 2010, 66, (2), 213-221. 
55. Emsley, P.; Cowtan, K., Coot: model-building tools for molecular graphics. Acta Crystallographica Section D 2004, 60, (12 Part 1), 2126-2132.

56. Davis, I. W.; Murray, L. W.; Richardson, J. S.; Richardson, D. C., MOLPROBITY: structure validation and all-atom contact analysis for nucleic acids and their complexes. Nucleic Acids Res 2004, 32, (Web Server issue), W615-9.

57. De Lano, W. The PyMOL Molecular Graphics System, 1.7.4.4.; Schrödinger, LLC.

58. Karplus, P. A.; Diederichs, K., Linking crystallographic model and data quality. Science 2012, 336, (6084), 1030-3. 
TABLE OF CONTENTS GRAPHIC.

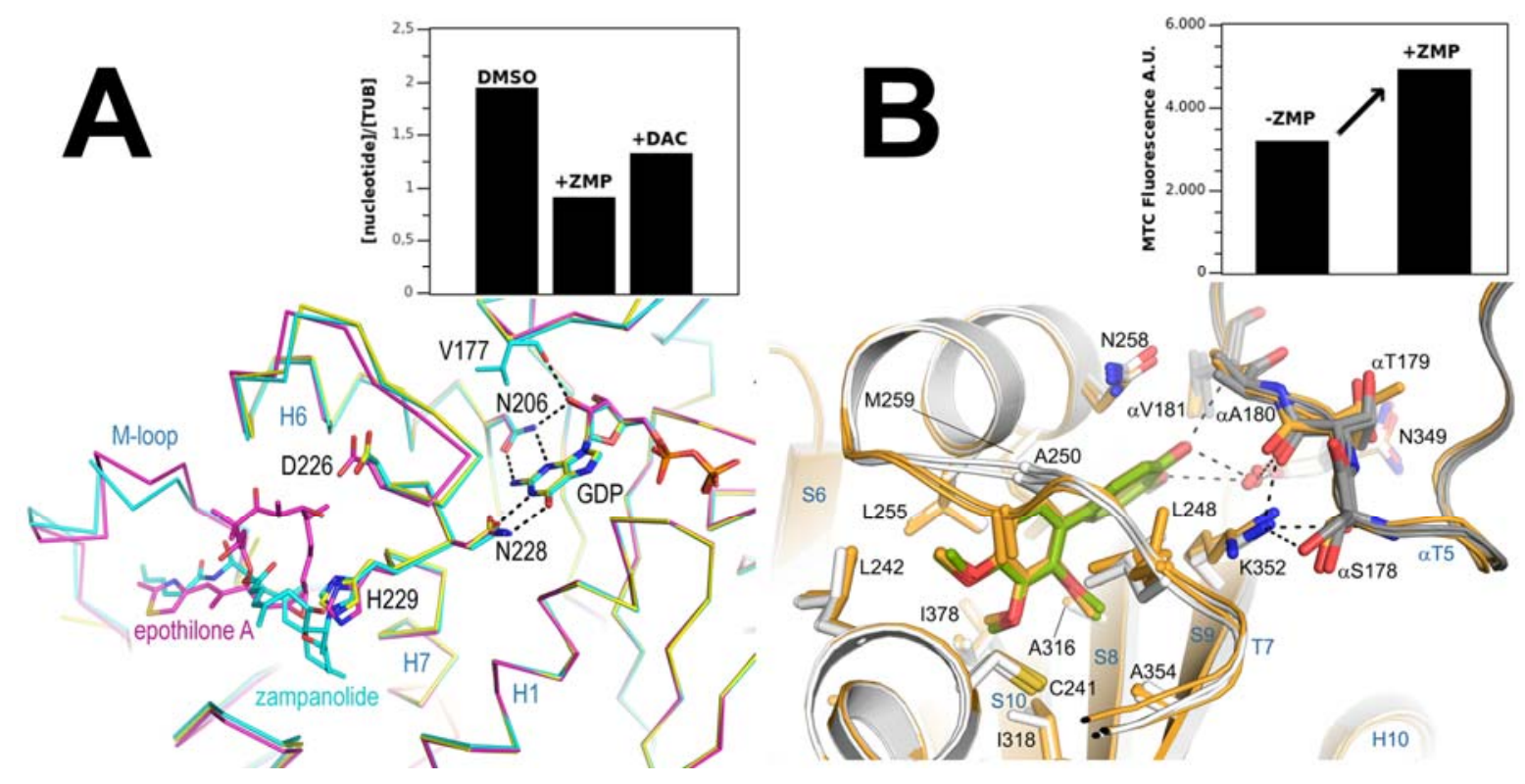

